\title{
Low Frequency Active Sonar Performance in the Arctic Beaufort Lens
}

\author{
by \\ Lieutenant Scott Adams Carper, USN
}

Submitted to the Joint Program in Applied Ocean Science \& Engineering and the Department of Electrical Engineering and Computer Science in partial fulfillment of the requirements for the degrees of

Master of Science in Oceanographic Engineering

and

Master of Science in Electrical Engineering and Computer Science Jointly by MIT-WHOI at the

\section{MASSACHUSETTS INSTITUTE OF TECHNOLOGY and the}

WOODS HOLE OCEANOGRAPHIC INSTITUTION

\section{September 2017 \\ (C)2017 Scott A. Carper.}

All rights reserved.

The author hereby grants to MIT and WHOI permission to reproduce and to distribute publicly paper and electronic copies of this thesis document in whole or in part in any medium now known or hereafter created.

Author

Joint Program in Applied Ocean Science \& Engineering Massachusetts Institute of Technology \& Woods Hole Oceanographic Institution August 18, 2017

Certified by

Henrik Schmidt

Professor of Mechanical and Ocean Engineering Thesis Supervisor

Certified by

Arthur B. Baggeroer Professor of Mechanical, Ocean, and Electrical Engineering Ford Professor of Engineering, Emeritus Thesis Supervisor

Accepted by . . Chair, Mechanical Engineering Committee for Graduate Students Quentin Berg Professor of Mechanics

Accepted by

Leslie A. Kolodziejski Chair, EECS Committee for Graduate Students Professor of Electrical Engineering

Accepted by

Henrik Schmidt Chair, Joint Committee for Applied Ocean Science and Engineering 


\title{
Low Frequency Active Sonar Performance in the Arctic Beaufort Lens
}

\author{
Scott A. Carper, LT, USN
}

Submitted to the Joint Program in Applied Ocean Science \& Engineering and the Department of Electrical Engineering and Computer Science on August 18th, 2017, in partial fulfillment of the requirements for the degrees of Master of Science in Oceanographic Engineering

and

Master of Science in Electrical Engineering and Computer Science Jointly by

MIT-WHOI

\begin{abstract}
A newly discovered double ducted acoustic environment present throughout much of the Beaufort Sea in the Arctic has a major effect on active acoustic transmissions. This work performs an in depth analysis of how the lower duct impacts the propagation of various active signals used commonly for acoustic communications or active sonar. First, this thesis performs a thorough modal analysis of the effect of the double ducted environment on long range propagation of a $300 \mathrm{~Hz}$ and $3500 \mathrm{~Hz}$ pulse. Signal excess is determined for the two different source pulses to quantify the effect of the lower duct on noise and SNR. Finally, channel capacity is calculated for the two frequency bands to evaluate operational impacts of the lower duct on acoustic communication systems in the Arctic.

Thesis Supervisor: Henrik Schmidt

Title: Professor of Mechanical and Ocean Engineering

Thesis Supervisor: Arthur Baggeroer

Title: Ford Professor of Engineering, Emeritus

Professor of Mechanical, Ocean, and Electrical Engineering
\end{abstract}




\section{Acknowledgments}

First I must thank the Navy for providing this fantastic opportunity to study at two of the most prestigious institutions in the world. Next, I owe many thanks to Professor Henrik Schmidt. Henrik welcomed me into his lab, brought me to the Arctic for a week, and provided countless hours of guidance and assistance. What I will remember most is his infectious optimism. Professor Arthur Baggeroer challenged me to make the most of my limited time at MIT. I could not have survived MIT without his encouragement and mentorship. Hopefully he is now able to spend more time enjoying his "retirement." To my fellow members of LAMSS, especially Nick, Thom, Rui, Kristen, Eesh, Erin, and Oscar, your help and friendship meant more to me than you know. To my classmates in the Joint Program, especially Jimmy, Jeffrey, Ryan, Lizzy, Chrissy, Daniel, Rachel, and Eric, I am truly humbled by your intelligence, work ethic, and most of all your enthusiasm. I look forward to following your careers as you change the world. Our ICEX-16 efforts would not have been possible without the support of Dr. Bob Headrick at ONR and Felix Rymsza and LCDR Justin Ivancic at the Undersea Warfare Development Center. Group 37 at Lincoln Laboratory did tremendous work analyzing the data from ICEX-16 and Dr. Jon Collis provided invaluable teaching and friendship in my summer there. There are far too many people at MIT and WHOI to thank each individually, but everyone I worked with at both institutions made this experience one I will never forget.

Finally, I must thank my family. My parents and my in-laws opened their doors to my wife and my son for almost a year which allowed Steph to pursue her professional goals and complete her Master's Degree. Both Steph and I are eternally grateful. Last but not least, I have to thank my incredible wife Steph and our amazing son Nicky for putting up with my long hours and high stress levels. Your love and encouragement got me through every tough day. 


\section{Contents}

1 Introduction $\quad 12$

1.1 Motivation . . . . . . . . . . . . . . . 12

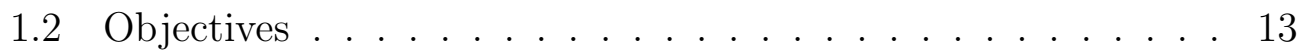

2 Background $\quad 14$

2.1 Classic Arctic Acoustic Environment . . . . . . . . . . . . . . 14

2.2 WHOI Ice-Tethered Profiler Program and the Beaufort Lens . 19

2.3 The Transarctic Acoustic Propagation

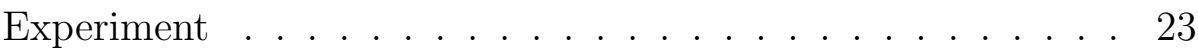

2.4 ICEX $2016 \ldots \ldots \ldots \ldots$

3 Analysis Methods $\quad 28$

3.1 Underwater Acoustics . . . . . . . . . . . . . . . . 28

3.1.1 Wavenumber Integration and OASES . . . . . . . . 30

3.1.2 Normal Mode Theory and KRAKEN . . . . . . . . . . 32

3.2 Signal Processing . . . . . . . . . . . . . . . . 34

3.2 .1 Active Sonar . . . . . . . . . . . . . . . . . 34

3.2.2 Matched Filtering . . . . . . . . . . . . . 38 
3.3 Channel Capacity . . . . . . . . . . . . . . . 41

4 Results 43

4.1 Modal Dispersion . . . . . . . . . . . . . . . . 43

$4.1 .1300 \mathrm{~Hz}$ Pulse ................... . . 43

$4.1 .23500 \mathrm{~Hz}$ Pulse . . . . . . . . . . . . . . 54

4.2 Signal Excess . . . . . . . . . . . . . . . . . . . 62

4.2.1 $300 \mathrm{~Hz}$ Pulse ................... 62

4.2.2 $3500 \mathrm{~Hz}$ Pulse . . . . . . . . . . . . . . . . . . . 69

4.3 Channel Capacity . . . . . . . . . . . . . . . 74

4.3.1 $300 \mathrm{~Hz}$ Pulse . . . . . . . . . . . . . . . 74

$4.3 .23500 \mathrm{~Hz}$ Pulse . . . . . . . . . . . . . . . . . . 78

5 Conclusion \& Future Work 82 


\section{List of Figures}

2.1 Comparison of Arctic SSP with an open ocean SSP $\ldots \ldots$.

2.2 Wenz Curves . . . . . . . . . . . . . . . . . . . . . . 18

2.3 Contrast between historical and new SSPs in the Arctic . . . . 20

2.4 Transmission loss example in the classic Arctic . . . . . . . . . 21

2.5 Transmission loss example in the new Arctic . . . . . . . . . 21

2.6 Spatial extent of the Beaufort Lens . . . . . . . . . . . 22

2.7 Example PSD of classic Arctic noise . . . . . . . . . . . . 24

2.8 ICEX ambient noise measurement experiment . . . . . . . 26

2.9 Power spectral density estimate of ambient noise at ICEX . . 27

3.1 Active sonar illustration . . . . . . . . . . . . . 34

$3.2300 \mathrm{~Hz}$ Source Pulse . . . . . . . . . . . . . . . . . . . . . 39

$3.33500 \mathrm{~Hz}$ Source Pulse . . . . . . . . . . . . . . . . . . . . 40

3.4 Active sonar block diagram . . . . . . . . . . . . 40

4.1 Mode shapes of the first 20 modes of a $300 \mathrm{~Hz}$ source pulse in a typical Arctic SSP . . . . . . . . . . . . . . . . 44

4.2 Modes shapes of the first 20 modes of a $300 \mathrm{~Hz}$ source pulse in the presence of the Beaufort Lens . . . . . . . . . . . . 45 
4.3 Phase and group speeds of ducted modes of a $300 \mathrm{~Hz}$ pulse . . 46

4.4 Filtered time series for a $300 \mathrm{~Hz}$ source in the surface duct in the presence of the Beaufort Lens . . . . . . . . . . . . 47

4.5 Range stacked plots for a $300 \mathrm{~Hz}$ source at 150 meters and a target at 30 meters with ice cover in the presence of the Beaufort Lens . . . . . . . . . . . . . . . . . . . . . 48

4.6 Filtered time series for a $300 \mathrm{~Hz}$ source in the surface duct in the presence of the Beaufort Lens . . . . . . . . . . . . . . 49

4.7 Filtered time series for a $300 \mathrm{~Hz}$ source in the lower duct as the target moves from the top of the duct to the bottom of

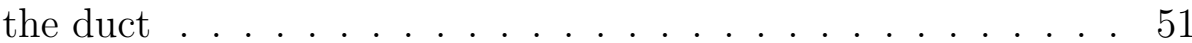

4.8 Range stacked time series for a source and a target in the Beaufort Lens . . . . . . . . . . . . . . . . . . 52

4.9 Depth stacked demodulated response as the target moves through the duct at 100 kilometers with the source at 150 meters in the Beaufort Lens with ice cover. . . . . . . . . . . . . 53

4.10 Mode shapes of the first 100 modes of a $3500 \mathrm{~Hz}$ source pulse in the presence of the Beaufort Lens. . . . . . . . . . . . . . 54

4.11 Range stacked time series for a $3500 \mathrm{~Hz}$ source and a target in the Beaufort Lens . . . . . . . . . . . . . . 56

4.12 Filtered response for active $3500 \mathrm{~Hz}$ sonar with both target and source in the lower duct at $150 \mathrm{~m} . \ldots . . . . . .557$

4.13 Range stacked response for a target in the surface duct at 30 meters with the $3500 \mathrm{~Hz}$ source in the lower duct at 150 meters 58 
4.14 Filtered response for a $3500 \mathrm{~Hz}$ pulse for a target at a range of 30 kilometers as it moves vertically through the duct. . . . . 60

4.15 Depth stacked demodulated response for a target in the duct at $30 \mathrm{~km}$ with a $3500 \mathrm{~Hz}$ source . . . . . . . . . . . . 61

4.16 Signal excess for a $300 \mathrm{~Hz}$ source at 30 meters in the classic Arctic with ice cover. . . . . . . . . . . . . . . 63

4.17 Signal excess for a $300 \mathrm{~Hz}$ source at 30 meters in the presence of the Beaufort Lens with ice cover . . . . . . . . . 63

4.18 Signal excess for a $300 \mathrm{~Hz}$ source at 30 meters in the presence of the Beaufort Lens with no ice cover . . . . . . . . . . . 64

4.19 Signal excess for a $300 \mathrm{~Hz}$ source at 150 meters in the presence of the Beaufort Lens with ice cover. . . . . . . . . . . . . 65

4.20 Signal excess difference for a $300 \mathrm{~Hz}$ source at 150 meters and at 30 meters in the new Arctic . . . . . . . . . . . . 66

4.21 Signal excess for a $300 \mathrm{~Hz}$ source at 150 meters in the new Arctic with only one-way propagation. . . . . . . . . 66

4.22 Noise power vs. depth for a $300 \mathrm{~Hz}$ source as predicted by

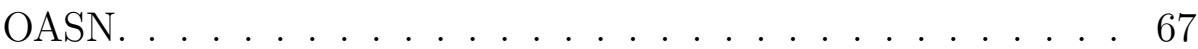

4.23 Signal excess for a $300 \mathrm{~Hz}$ source at 150 meters in the new Arctic with depth-dependent noise. . . . . . . . . . . . . . . 68

4.24 Signal excess for a $3500 \mathrm{~Hz}$ source at 30 meters in the classic Arctic with smooth ice cover. . . . . . . . . . . . . . 70

4.25 Signal excess for a $3500 \mathrm{~Hz}$ source at 30 meters in the presence of the Beaufort Lens with smooth ice cover . . . . . . . . 70 
4.26 Signal excess in the new Arctic for a deep $3500 \mathrm{~Hz}$ source with smooth ice cover . . . . . . . . . . . . . . 71

4.27 Signal excess difference between a $3500 \mathrm{~Hz}$ source at 30 meters and 150 meters in the presence of the Beaufort Lens with smooth ice cover. . . . . . . . . . . . . . . . 72

4.28 Noise power vs. depth for a $3500 \mathrm{~Hz}$ source as predicted by

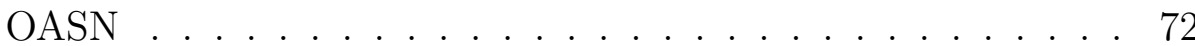

4.29 Signal excess for a $3500 \mathrm{~Hz}$ source with depth dependent noise 73

4.30 Historic Arctic channel capacity for a $300 \mathrm{~Hz}$ source at 30 meters with ice cover . . . . . . . . . . . . . . 74

4.31 Channel capacity estimate using the new Arctic SSP with a $300 \mathrm{~Hz}$ source at 30 meters and ice cover . . . . . . . . . . . . 75

4.32 Channel capacity estimate using the new Arctic SSP with a $300 \mathrm{~Hz}$ source at 150 meters and ice cover . . . . . . . . . . 76

4.33 Channel capacity difference in the new Arctic with a $300 \mathrm{~Hz}$ source in the lower duct vs a source in the surface duct with ice cover . . . . . . . . . . . . . . . . . 77

4.34 Channel capacity estimate for a $3500 \mathrm{~Hz}$ source at 30 meters in the classic Arctic with smooth ice cover . . . . . . . . . 78

4.35 Channel capacity estimate for a $3500 \mathrm{~Hz}$ source at 30 meters in the presence of the Beaufort Lens and smooth ice cover . . 79

4.36 Channel capacity estimate for a $3500 \mathrm{~Hz}$ source at 150 meters in the Beaufort Lens and smooth ice cover. . . . . . . . . . . . 80 
4.37 Channel capacity ratio of a $3500 \mathrm{~Hz}$ source in the lower duct vs. that when the source is placed in the surface duct in the new Arctic . . . . . . . . . . . . . . . . . . . . . . . 81 


\section{Chapter 1}

\section{Introduction}

\subsection{Motivation}

Global interest in the Arctic is increasing rapidly as new technology and climate change allow access to resources that were previously inaccessible, and open shipping lanes that had been frozen for all or most of the year in past decades. Submarines and autonomous underwater vehicles (AUVs) serve as one of the primary means of exploration due to their ability to operate remotely under the ice cover; however, they rely heavily on sonar and acoustic transmissions for navigation and communication. Recent environmental changes have dramatically impacted underwater acoustic propagation in regions of the western Arctic, most notably in the Beaufort Sea. A neutrally buoyant layer of warm water from the North Pacific has entered the Beaufort Sea through the Bering Strait. This layer of water, called the "Beaufort Lens," creates a double-ducted acoustic environment [2]. If ignored, these changes will significantly impact current operating capabilities 
in the Arctic Ocean.

However, a thorough understanding of the new environment's effects on active transmissions can be used to lower transmission loss (TL) and greatly enhance effective ranges of active transmissions, improving capabilities of AUVs and submarines operating in the Arctic.

\subsection{Objectives}

This thesis presents a thorough analysis of the impact of the double ducted acoustic environment on several commonly used active acoustic signals. It provides an in-depth analysis of the modal propagation of various narrowband signals and then develops a matched filter signal processing

scheme. It performs an analysis of the channel capacities of the classical and double-ducted Arctic acoustic environments for acoustic communications applications. Finally, it shows how this matched filter scheme can be used to maximize signal excess at a receiver which can be used to enhance autonomy or alter operating patterns to leverage the new duct. 


\section{Chapter 2}

\section{Background}

\subsection{Classic Arctic Acoustic Environment}

The major constraints of underwater active acoustic transmissions are signal-to-noise ratio (SNR), attenuation, Doppler, multipath propagation, spreading, and clutter from bottom boundary reverberation. Multipath propagation is one of the most complicated effects to resolve in underwater acoustic applications. As the sound wave travels, it reflects off the surface and bottom boundaries, and refracts towards lower sound speeds in accordance with Snell's law. The result is that a receiver will receive numerous reflected/refracted paths that all originated as the same signal.

Acoustic wave refraction is a result of the varying speed of sound in water. Equation 2.1 shows the speed of sound as a function of temperature 
$\left(\mathrm{T}\left({ }^{\circ} \mathrm{C}\right)\right)$, depth $(\mathrm{z}(\mathrm{m}))$, and salinity $(\mathrm{S}(\mathrm{ppt}))[1]$.

$c(z, T, S)=1449.2+4.6 T-0.055 T^{2}+0.00029 T^{3}+(1.34-0.01 T)(S-35)+0.016 z$

Throughout most of the ocean, the speed of sound is dominated by temperature effects for the first 1000 meters. The rate of change of the sound speed with respect to temperature varies significantly with water temperature. For example, in very cold Arctic water, $\frac{d c}{d T} \approx 4.6 \frac{\mathrm{m} / \mathrm{s}}{{ }^{\circ} \mathrm{C}}$, while at $20^{\circ} \mathrm{C}, \frac{d c}{d T} \approx 2.4 \frac{\mathrm{m} / \mathrm{s}}{{ }^{\circ} \mathrm{C}}$. Deeper than 1000 meters, the ocean is isothermal for the most part and thus the effect of hydrostatic pressure, incorporated in equation 2.1 as a depth term, dominates. In the open ocean, the salinity term does not have a major impact on spatial variation of sound speed except at interfaces where large bodies of water mix such as the sill of Gibraltar. However, in the Arctic, the halocline effects can be significant in the first 100 meters because of the freshwater from the ice cover.

The Arctic creates an acoustic environment that is unique to the rest of the world. The polar ice cap reflects the sunlight and maintains a relatively isothermal water column. The net effect results in a monotonically increasing sound speed with depth. Figure 2.1 contrasts the classical Arctic sound speed profile (SSP) with a typical SSP from the open ocean [1]. The upward refracting SSP bends acoustic waves back towards the surface creating what is known as a surface duct. The surface duct results in numerous lossy interactions between the ice and the propagating wave. Thus, acoustic wave propagation in a traditional Arctic SSP is significantly limited by the ice interactions. A typical transmission loss plot in the classic Arctic in figure 


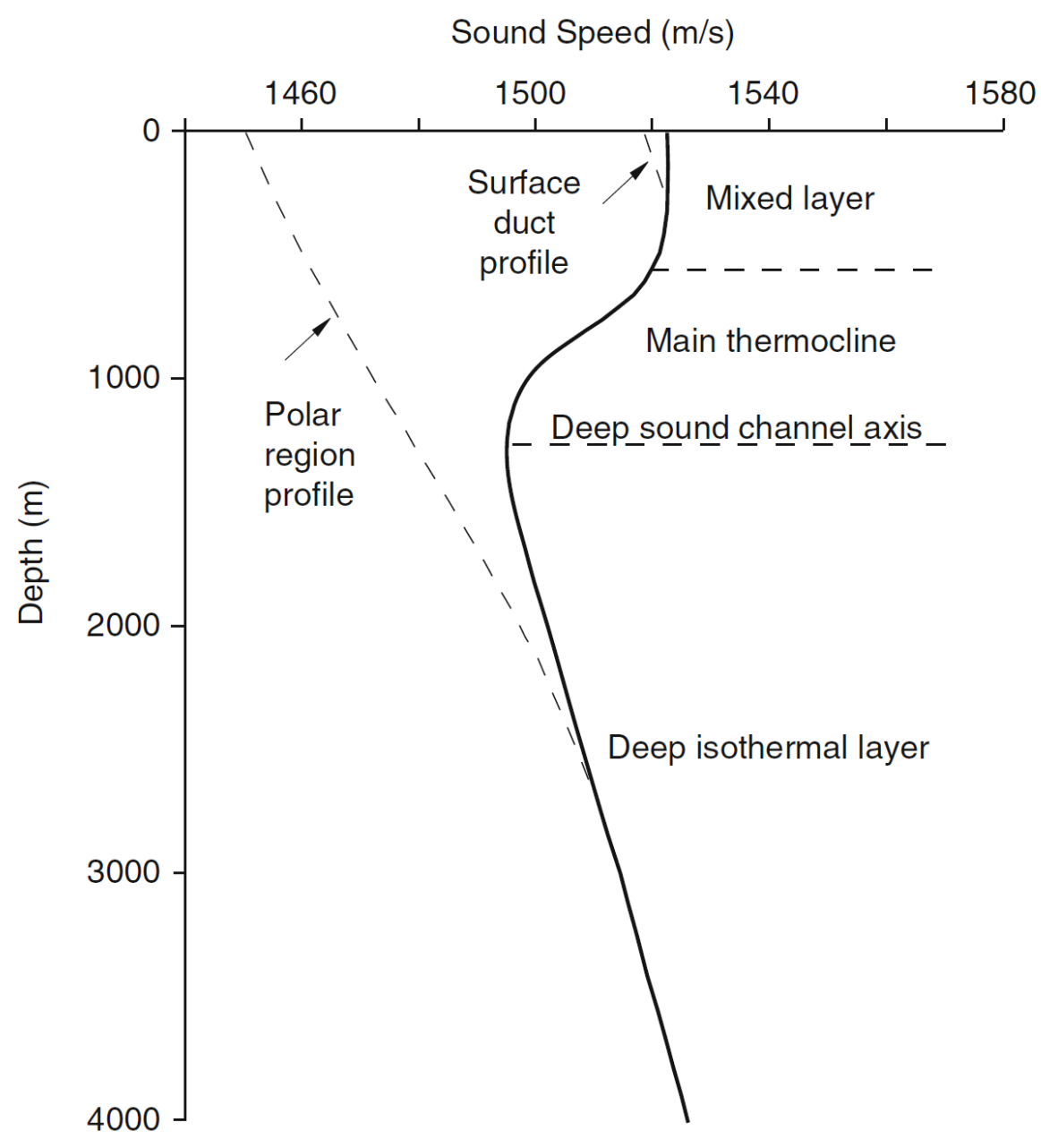

Figure 2.1: A comparison of a notional Arctic SSP with a typical open ocean SSP [1]. This figure is provided to show the general shape of the SSP that you would expect to see in the Arctic (dashed line) and the deep ocean (solid line). Neither SSP shown is an exact representation of any location. 


\section{4 illustrates this effect.}

While the upward refracting SSP of the classic Arctic limits the range of acoustic propagation, it also serves to significantly reduce the ambient acoustic noise if there is low ice activity. Any surface noise is quickly attenuated as the ice interactions scatter the acoustic waves. Furthermore, a large component of ambient noise in the ocean is created by surface vessels, waves, and geological surveying, all of which are currently mostly precluded by the Arctic ice cover. These two effects create a region of very low ambient noise in the Arctic when the ice is not active. The Wenz curves in figure 2.2 show how shipping and surface noise usually dominate the ambient noise field [9]. 


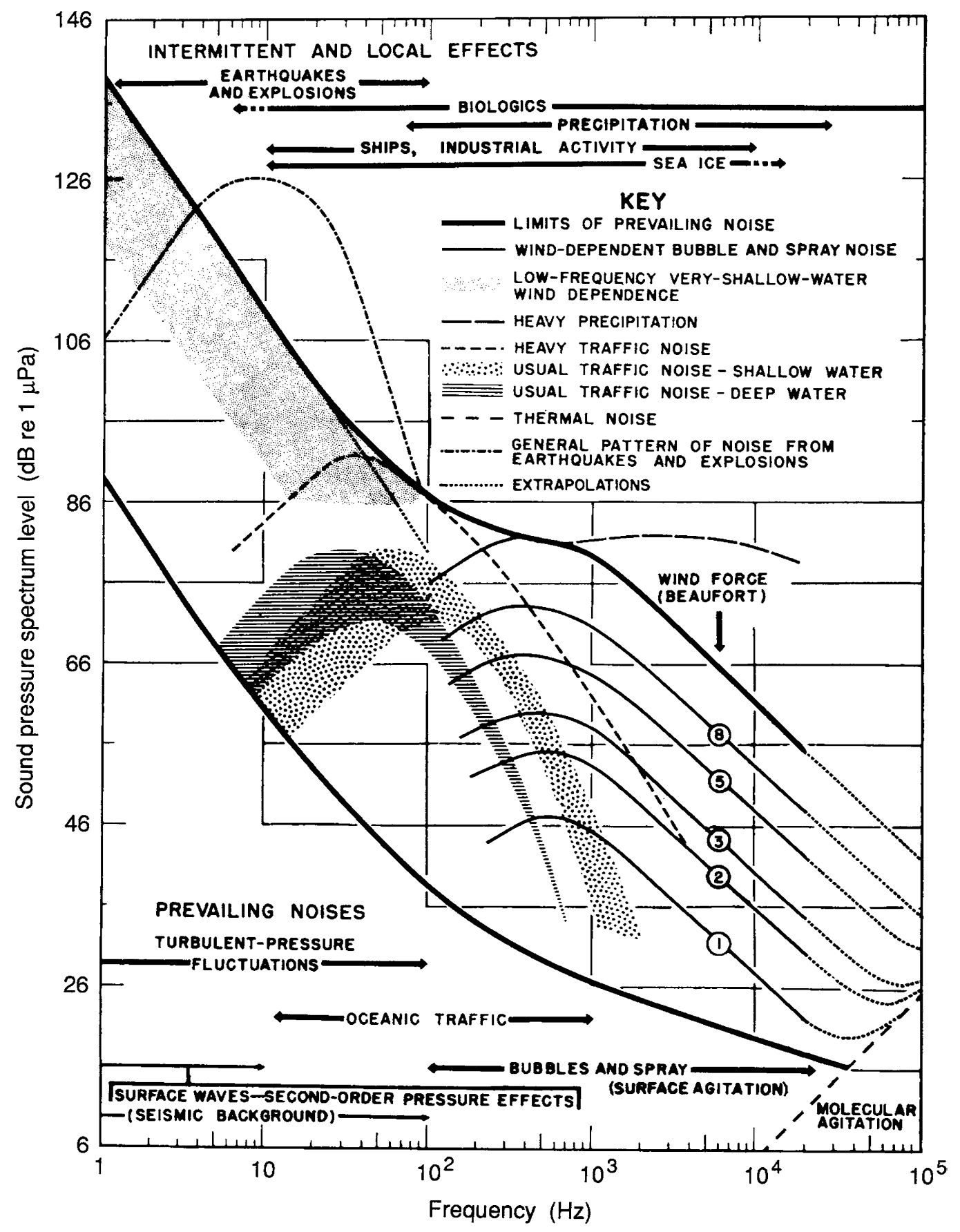

Figure 2.2: The Wenz Curves show empirically derived ambient noise levels and spectra for sources of noise in the ocean. [9] 


\subsection{WHOI Ice-Tethered Profiler Program and the Beaufort Lens}

Over the past 20 years, and perhaps even earlier, a warm water layer referred to as the "Beaufort Lens"[2] has been discovered and studied in parts of the Beaufort Sea off of the northern coast of Alaska. In 2004, the Woods Hole Ice Tethered Profiler (ITP) program detected a warm, neutrally buoyant, layer of water entering the Arctic Ocean from the Northern Pacific. The Ice-Tethered Profiler data were collected and made available by the IceTethered Profiler Program (Toole et al., 2011; Krishfield et al., 2008) based at the Woods Hole Oceanographic Institution (http://www.whoi.edu/itp) [4]. The warm layer of water exists between 80-100 meters and creates a local maximum in the SSP around 90 meters. This peak in the sound speed forms a second duct, below the surface duct, where sound can travel relatively long distances without any lossy ice or bottom interactions. The Lens ducts the sound resulting in cylindrical vice spherical spreading, further enhancing long distance propagation.

As discussed previously, the Arctic often has very low ambient noise. Most noise created by ice events is trapped in the upper surface duct. The lower duct has very low noise and a mechanism for sound that propagates by refraction only which enables high signal-to-noise ratios at long ranges. This exciting potential is the inspiration for this thesis. A representative SSP from the Beaufort lens is shown in contrast with an SSP exhibiting the typical Arctic characteristics in figure 2.3. Figure 2.5 shows the same source as figure 2.4, but in the new Arctic environment. The transmission loss 
plot highlights the potential for long range propagation. These SSPs were calculated using equation 2.1 and data from the Woods Hole Oceanographic Institution's (WHOI) ITP Program.

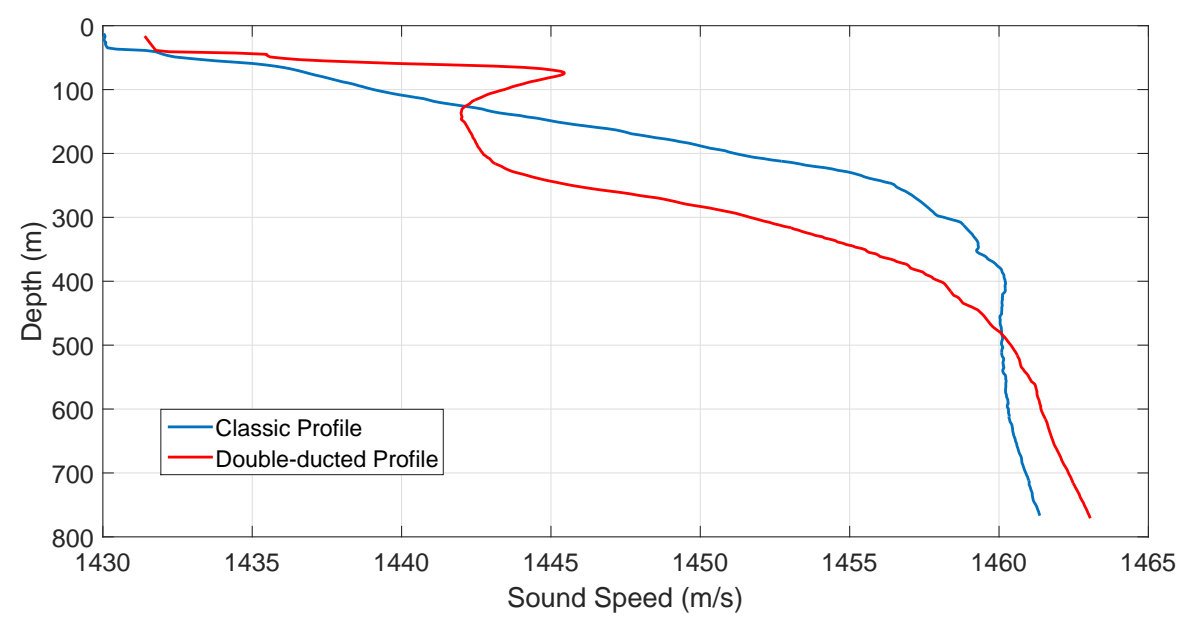

Figure 2.3: A comparison of the double-ducted SSP with the historical upward refracting SSP. In the classic Arctic SSP, the first 100 meters are the halocline where the sound speed is dominated by the effect of salinity. Below 100 meters is the pycnocline where the increasing density of the water dominates the overall sound speed since temperature and salinity are relatively constant. In the Beaufort Lens, the warm water layer causes temperature to be the dominant factor in sound speed between about 100 and 300 meters, interrupting the halocline that was seen traditionally.

The spatial and temporal extent of the Beaufort Lens, as well as its variability, is examined in depth in [3]. Figure 2.6 shows the spatial extent of the double-ducted SSP as of 2015. The temporal variation is shown to be insignificant and the thesis by Howe concludes that the Beaufort Lens is a "persistent phenomenon" [3]. The Beaufort Lens exists throughout most of the Beaufort Sea which is an area of significant commercial and military interest for the United States. This thesis examines the possibility of ex- 


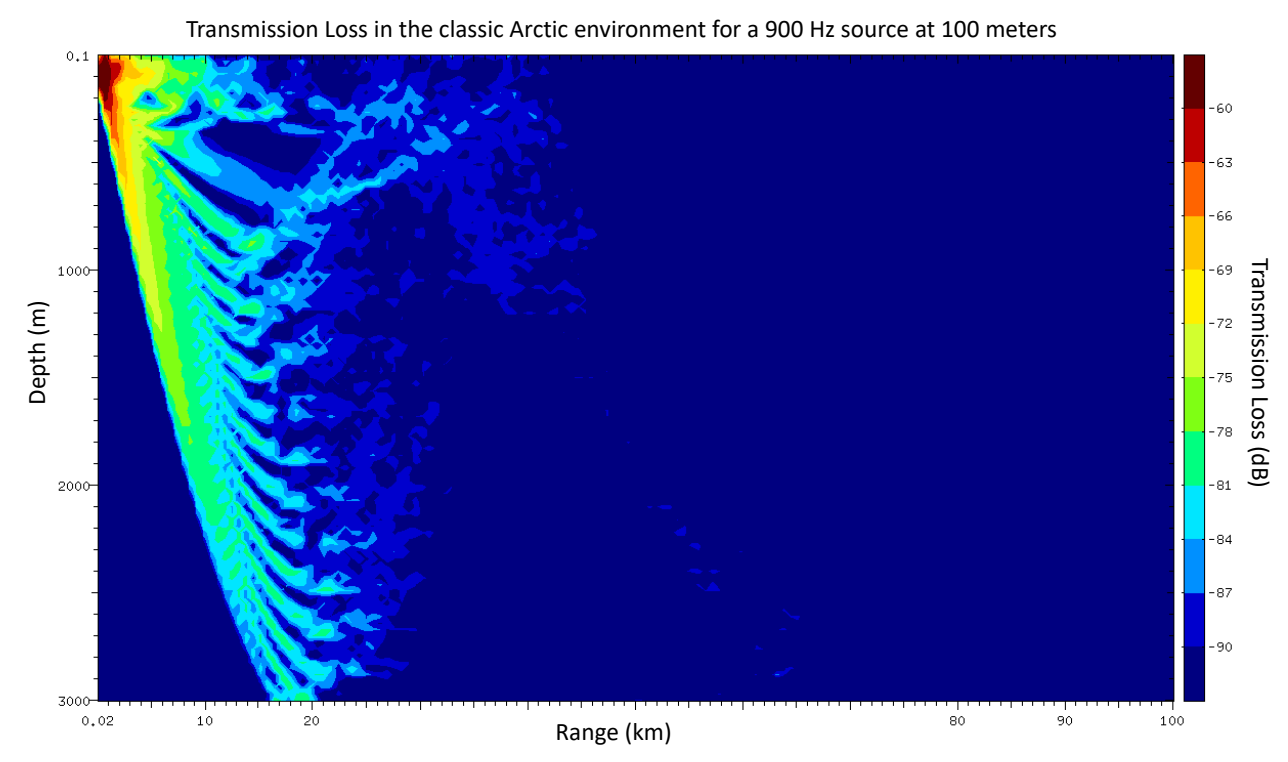

Figure 2.4: Transmission loss plot of a $900 \mathrm{~Hz}$ source at 100 meters in the classic Arctic environment

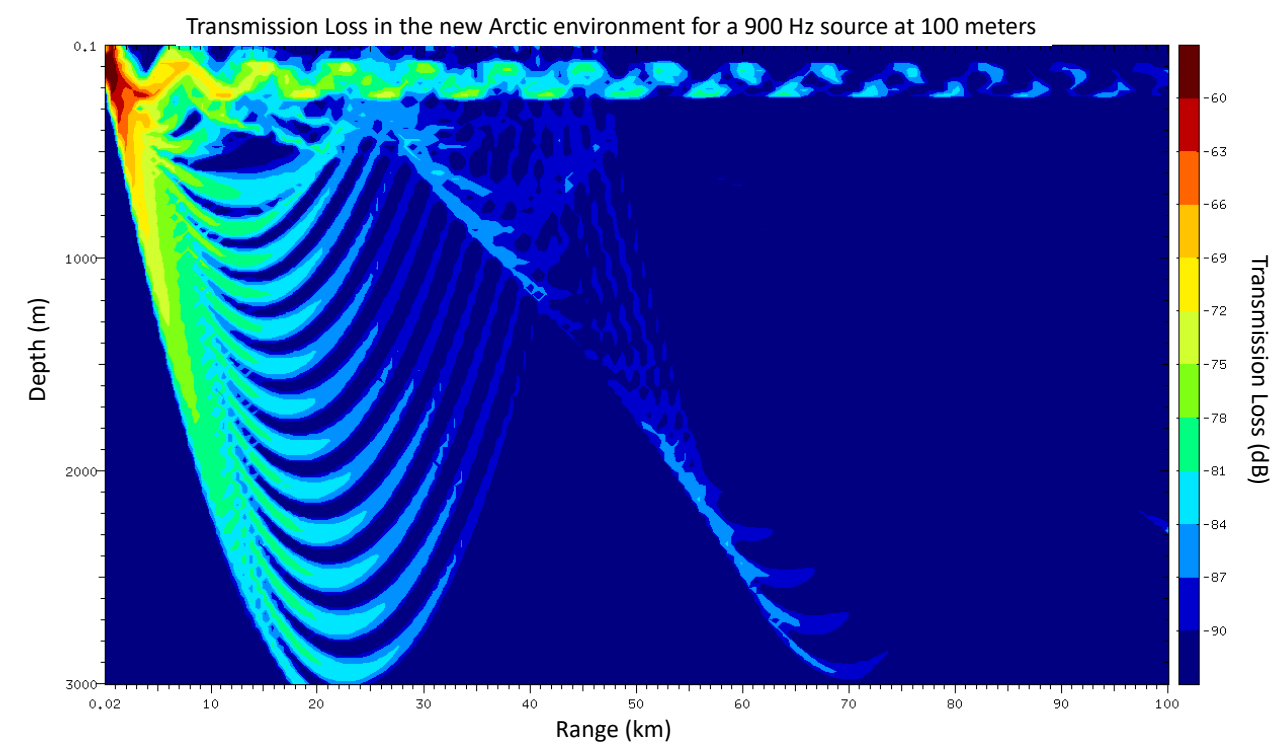

Figure 2.5: Transmission loss plot of a $900 \mathrm{~Hz}$ source at 100 meters in the new Arctic environment. 
ploiting the Beaufort Lens and focuses on how it can be used to enable long range acoustic transmissions for communication and navigation.

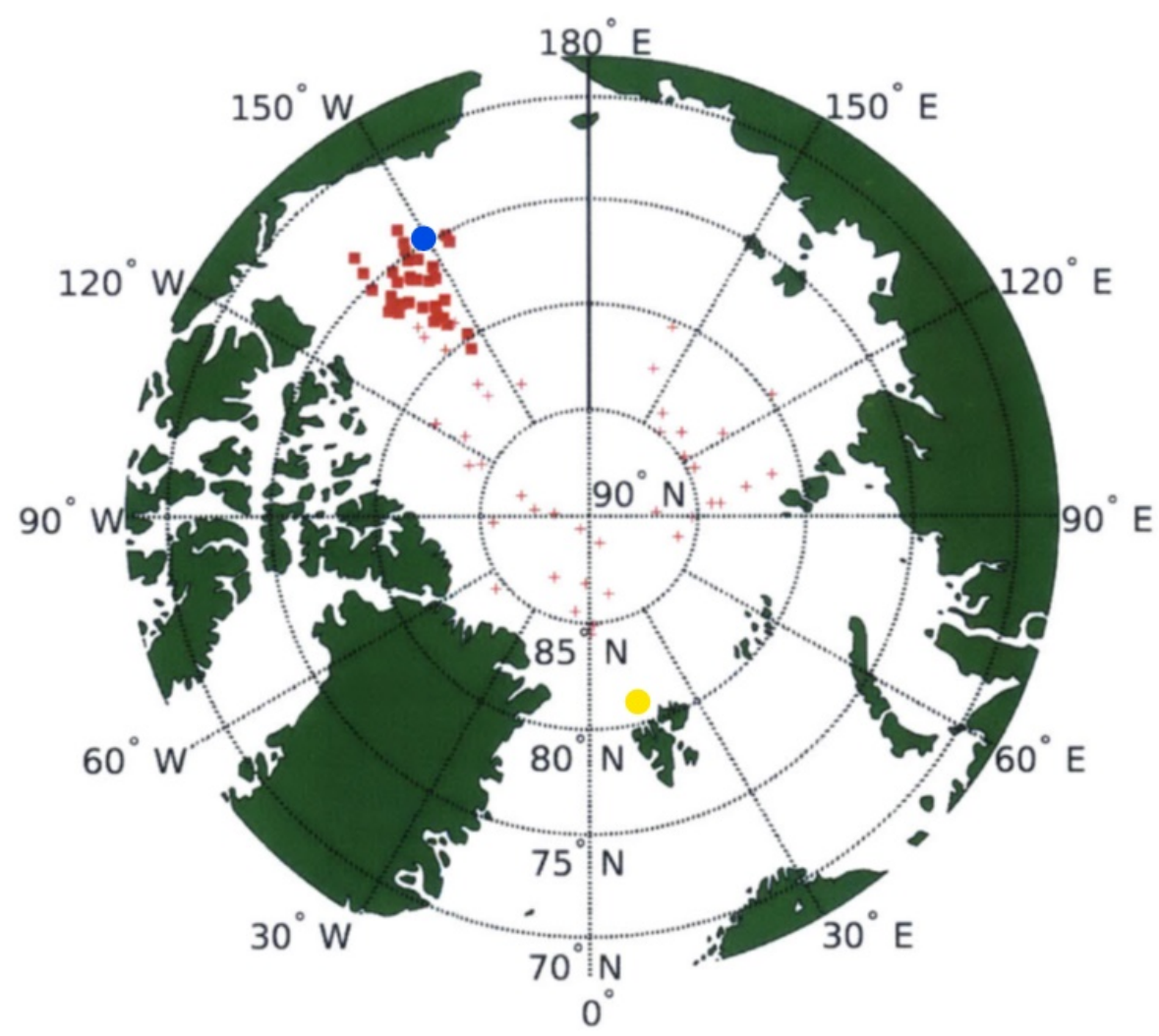

Figure 2.6: The spatial extent of the Beaufort Lens [3]. The double ducted profiles were detected by ITPs marked with red boxes. A red ' + ' represents an ITP that has not detected the Beaufort Lens. The blue dot shows the approximate locations of the ICEX and TAP camps. The yellow dot shows the approximate location of the source used for the TAP experiment. 


\subsection{The Transarctic Acoustic Propagation Experiment}

In April of 1994 the Transarctic Acoustic Propagation (TAP) Experiment was conducted in an effort to demonstrate the feasibility of acoustic tomography in the Arctic [5]. A 32-element vertical line array (VLA) as well as a 32-element horizontal array were used to collect acoustic data from a source approximately 2600 kilometers away. The source was just north of Svalbard, Norway. The TAP camp was in the Beaufort Sea in a similar location to the ICEX-16 camp. The receiver for TAP was at the ice station SIMI (Sea Ice Mechanics Initiative). Both the source and receiver camps were supported by the Office of Naval Research (ONR) and their locations are shown in figure 2.6. The VLA ambient noise data will be used in this work because the environment is assumed to be two dimensional with a range independent SSP that varies only with depth. The VLA used for the TAP experiment had elements spaced seven meters apart ranging between 62 and 279 meters. The array was sampled at $1 \mathrm{kHz}$. A typical power spectral density (PSD) of the noise measured by one element of the array is shown in figure 2.7. The TAP data allows comparison between historic Arctic ambient noise and the new ambient noise field, but it is limited to low frequencies since the array was only sampled at $1 \mathrm{kHz}$. 


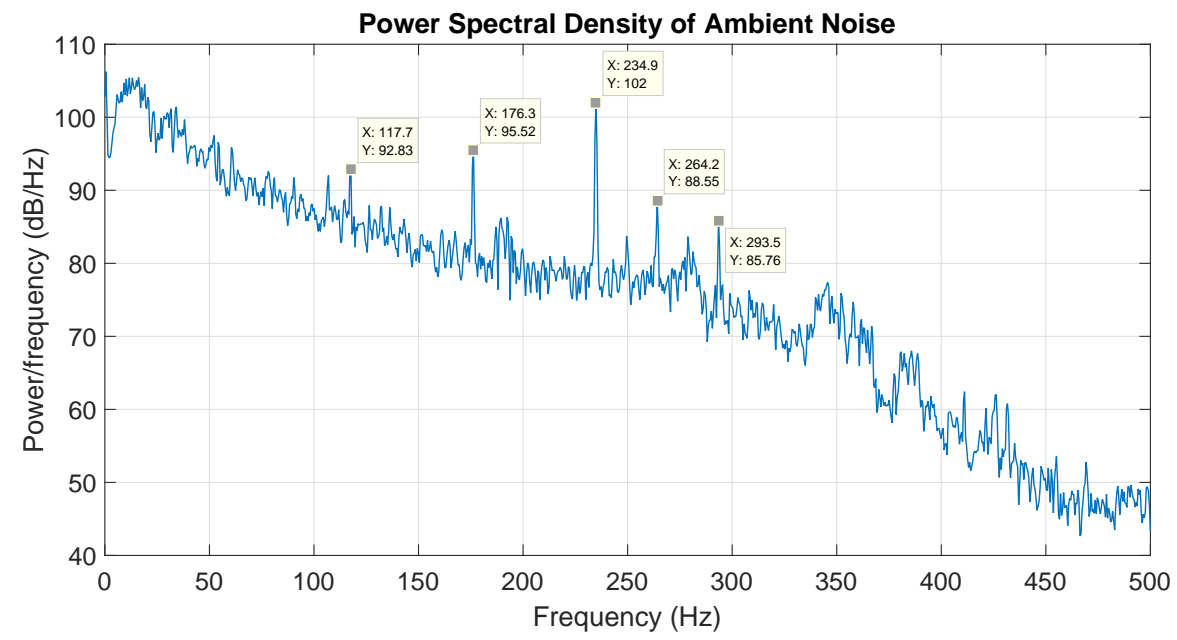

Figure 2.7: A typical power spectral density estimate of ambient Arctic noise from one element in the VLA used in the TAP experiment. The large peaks are $60 \mathrm{~Hz}$ harmonics caused by the electronic noise of the array and a $30 \mathrm{~Hz}$ generator operating at $1800 \mathrm{rpm}$. The peak at $270 \mathrm{~Hz}$ is mechanical noise from the generator. 


\section{$2.4 \quad$ ICEX 2016}

In March of 2016, the United States Navy staged ICEX 2016, a scientific and military exercise to develop operating capabilities in the Arctic (in the same region of the Arctic where the TAP experiment was conducted 22 years prior). Scientists from MITs Laboratory for Autonomous Marine Sensing Systems participated and spent 10 days on the ice floe deploying an autonomous underwater vehicle (AUV). The goal of the MIT study was to further characterize the Beaufort Lens, while simultaneously demonstrating Arctic operations of a large AUV. Even operating at relatively short ranges (1-6 km), the acoustic environment made tracking and communicating with the AUV very difficult. The reason for tracking difficulties is highlighted in section 4.2.2.

Acoustic data were collected using the towed hydrophone array of the vehicle as a vertical line array (VLA) suspended in the water column. During this portion of the experiment the vehicle was suspended by a tether, with its array weighted and suspended below it vertically. Starting at 25 meters, the vehicle was lowered in increments of 25 meters every 15 minutes to a maximum depth of 200 meters. This work uses the data collected in this ambient noise measurement experiment to provide a depth dependent characterization of Arctic ambient noise in the Beaufort Sea. An estimate of the PSD from one element of the VLA is shown in figure 2.9.

In addition to the MIT data collection, two American submarines, the USS Hartford and the USS Hampton, performed several tests to characterize the spatial variability of the SSP by collecting acoustic data and attempting to track each other over long ranges. Again, tracking and communications 


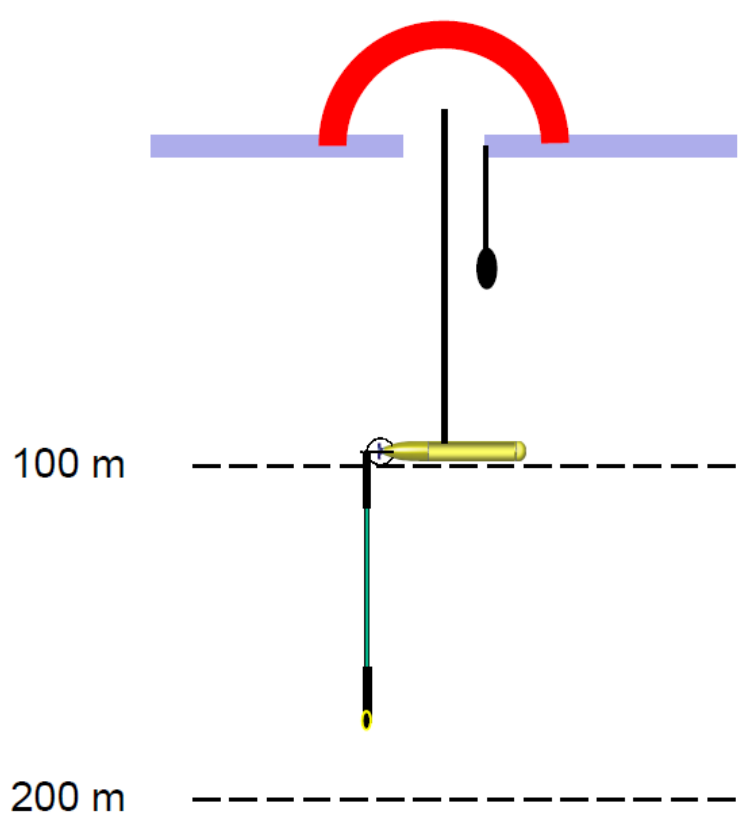

Figure 2.8: The ambient noise measurement experiment during ICEX-16 involved suspending the AUV from the ice and allowing its linear array to hang vertically below it. In this diagram the red semi-circle represents the tent on the ice. The vehicle was suspended at various depths to measure the noise both inside and outside of the duct. 


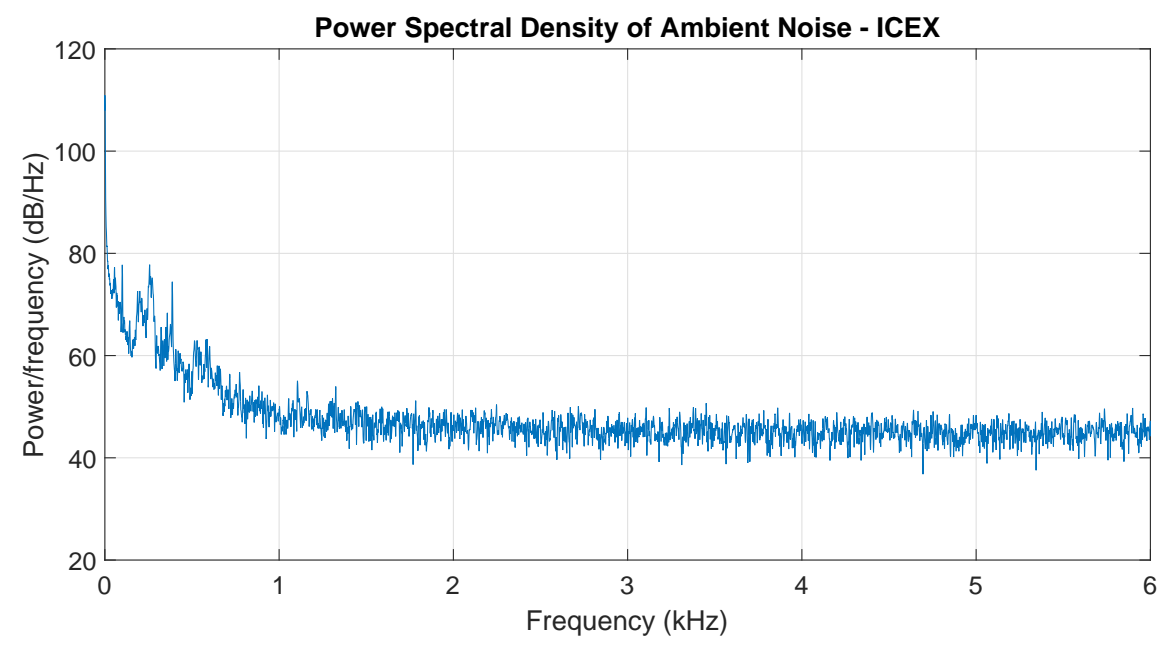

Figure 2.9: A typical power spectral density estimate of ambient Arctic noise from one element in the VLA used in the ICEX-16 experiment.

were significantly complicated by the acoustic environment. The data collected by the submarines were classified and are not used in this thesis. 


\section{Chapter 3}

\section{Analysis Methods}

\subsection{Underwater Acoustics}

Sound propagates through a medium the same way light propagates: as waves, just at a much lower speed. Underwater acoustic wave propagation is governed by the wave equation which can be linearly approximated as shown below. The following derivations are standard reference material and included to establish notation [1], [6].

$$
\begin{gathered}
\frac{\delta \rho^{\prime}}{\delta t}=-\nabla\left(\rho_{0} \vec{v}\right) \\
\frac{\delta \vec{v}}{\delta t}=-\frac{1}{\rho_{0}} \nabla p^{\prime}(\rho) \\
\frac{\delta p^{\prime}}{\delta t}=c^{2}\left(\frac{\delta \rho^{\prime}}{\delta t}+\vec{v} \nabla \rho_{0}\right)
\end{gathered}
$$

Here, $p$ and $\rho$ represent pressure and density respectively; $c$ and $\vec{v}$ represent sound speed and particle velocity. $p^{\prime}$ and $\rho^{\prime}$ are small perturbations in 
pressure and density and $\rho_{0}$ represents the steady state density. Equations 3.1-3.3 can be used to solve for the wave equation for pressure and the wave equation for particle velocity shown in Equations 3.4 and 3.5 respectively.

$$
\begin{gathered}
\nabla^{2} p-\frac{1}{c^{2}} \frac{\delta^{2} p}{\delta t^{2}}=0 \\
\frac{1}{\rho} \nabla\left(\rho c^{2} \nabla \vec{v}\right)-\frac{\delta^{2} \vec{v}}{\delta t^{2}}=0
\end{gathered}
$$

Assuming the density to be constant within a discrete layer model, and using the fact that velocity is the time derivative of displacement, $\vec{u}$, the displacement potential, $\psi$, is defined as:

$$
\vec{u}=\nabla \psi
$$

Using the impedance relationship for plane-waves in equation 3.7, the wave equation for pressure is re-written as shown in equation 3.8, where a source term has been added as a volume injection as a function of space and time.

$$
\begin{gathered}
\frac{p}{v_{x}}=\rho_{0} c \\
\nabla^{2} \psi-\frac{1}{c^{2}} \frac{\delta^{2} \psi}{\delta t^{2}}=f(\vec{r}, t)
\end{gathered}
$$

Simply taking the Fourier transform of equation 3.8 yields the frequencydomain wave equation known as the Helmholtz equation.

$$
\left[\nabla^{2}+k^{2}(\vec{r})\right] \psi(\vec{r}, \omega)=f(\vec{r}, \omega)
$$


Here, $\vec{k}(\vec{r})$ represents the medium wavenumber defined as:

$$
\vec{k}(\vec{r})=\frac{\omega}{c(\vec{r})}
$$

Most numerical approaches solve the Helmholtz equation instead of the time domain equations. This thesis uses a wavenumber integration technique as well as a normal modes approach which are explained in sections 3.1.1 and 3.1.2.

\subsubsection{Wavenumber Integration and OASES}

One numerical approach to solve the wave equation given in equation 3.9 is wavenumber integration. Wavenumber integration techniques reduce the Helmholtz equation to a set of one-dimensional differential equations in the depth dimension. The water column is divided into layers where each ordinary differential equation can be solved analytically. To derive the depthseparated wave equation (3.12), apply the forward Hankel transform (3.11) to the Helmholtz equation (3.9).

$$
\begin{gathered}
f\left(k_{r}, z\right)=\int_{0}^{\infty} f(r, z) J_{0}\left(k_{r} r\right) r d r \\
{\left[\frac{d^{2}}{d z^{2}}-\left[k_{r}^{2}-k_{m}^{2}(z)\right]\right] \Psi_{m}\left(k_{r}, z\right)=\frac{f_{s}(z)}{2 \pi}}
\end{gathered}
$$

The Hankel transform represents the decomposition of the wave into converging and diverging conical waves as shown:

$$
J_{0}\left(k_{r} r\right)=\frac{1}{2}\left[H_{0}^{(1)}\left(k_{r} r\right)+H_{0}^{(2)}\left(k_{r} r\right)\right]=\operatorname{Re}\left[H_{0}^{(1)}\right]
$$


Here the asymptotic Hankel functions are approximated as $k r \rightarrow \infty$ as:

$$
\begin{aligned}
H_{0}^{(1)}\left(k_{r} r\right) & \approx \sqrt{\frac{2}{\pi k_{r} r}} e^{j\left(k_{r} r-\pi / 4\right)} \\
H_{0}^{(2)}\left(k_{r} r\right) & \approx \sqrt{\frac{2}{\pi k_{r} r}} e^{-j\left(k_{r} r-\pi / 4\right)}
\end{aligned}
$$

The far field approximations shown above generally work well as long as $k r>3$. To move from the wavenumber domain back to the displacement and stress domain, the inverse integral transforms are applied using Fast Field Programs.

For this work, OASES, a commercially available wavenumber integration software is used to solve the depth separated wave equation (3.12) to model acoustic propagation in the Arctic. OAST, the transmission loss module is used to generate transmission loss plots [10, p. 33]. OASP, the wideband transfer function module, is used to return the acoustic channel transfer function [10, p. 59]. OASP returns the transfer function coefficients, $\alpha_{f_{i}}$, so that the channel transfer function can be assembled using equation 3.16 .

$$
h(t)=\Sigma \alpha_{f_{i}} e^{j 2 \pi f_{i} t}
$$

Finally, the OASN module is used to model Arctic ambient noise in the frequency bands of interest [10, p.93]. 


\subsubsection{Normal Mode Theory and KRAKEN}

Normal mode theory is one of several techniques commonly used to solve the wave equation when modeling underwater acoustic propagation. Once again, these derivations are standard reference material and included to establish notation [1], [6], [7]. Normal mode theory decomposes the Helmholtz equation into independent functions of depth and range such that $p(r, z)=\Phi(r) \Psi(z)$ as shown below:

$$
\frac{1}{\Phi}\left[\frac{1}{r} \frac{d}{d r}\left(r \frac{d \Phi}{d r}\right)\right]+\frac{1}{\Psi}\left[\rho(z) \frac{d}{d z}\left(\frac{1}{\rho(z)} \frac{d \Psi}{d z}\right)+\frac{\omega^{2}}{c^{2}(z)} \Psi\right]=0
$$

The only way this equation can be satisfied is if the depth dependent component and range dependent component are both equal to a constant, denoted $k_{r m}^{2}$. Thus, the modal equation, 3.18, is obtained.

$$
\rho(z) \frac{d}{d z}\left[\frac{1}{\rho(z)} \frac{d \Psi_{m}(z)}{d z}\right]+\left[\frac{\omega^{2}}{c^{2}(z)}-k_{r m}^{2}\right] \Psi_{m}(z)=0
$$

In this equation and those following, the $m$ indicates the mode or eigenvalue index. Solving this Sturm-Liouville eigenvalue problem, the pressure field is obtained by summing the solution for every mode:

$$
p(r, z)=\operatorname{Re}\left[\frac{j}{4 \rho\left(z_{s}\right)} \sum_{m=1}^{\infty} \Psi_{m}\left(z_{s}\right) \Psi_{m}(z) H_{0}^{(1)}\left(k_{r m} r\right)\right]
$$

While wavenumber integration already presents the exact solution, normal modes can produce excellent approximations quickly by only summing over the dominant modes. Normal modes theory is also useful in that individual 
modes can be examined to understand the dispersive effects of the waveguide. Using normal mode theory, it is easy to determine mode shape, phase velocity, and group velocity so that complicated, dispersed multi-path arrivals can be deciphered. For a given mode, the phase velocity, $v_{m}$, and group velocity, $u_{m}$, are calculated using equations 3.20 and 3.21 .

$$
\begin{gathered}
v_{m}=\frac{\omega}{k_{r m}} \\
u_{m}=\frac{d \omega}{d k_{r m}}
\end{gathered}
$$

Phase and group slowness are commonly used in literature as the mathematical derivation works out more cleanly and are simply defined as the inverse of phase and group speeds as shown below.

$$
\begin{gathered}
s_{p, m}=\frac{k_{r m}}{\omega} \\
s_{g, m}=\frac{d k_{r m}}{d \omega}
\end{gathered}
$$

A much more thorough explanation and derivation of normal mode theory can be found in [1].

This thesis uses KRAKEN to investigate which modes are trapped in the lower duct and thus which modes are the dominant modes at long ranges. KRAKEN is a publicly available normal modes code that can be downloaded with the Acoustics Toolbox [11], [12]. Like OASES, KRAKEN takes an input file containing the environmental and source parameters. KRAKEN outputs the number of propagating modes in a given environment, and their phase and group velocities. 
At long ranges, only the ducted modes significantly contribute to the acoustic pressure field since all other modes undergo numerous surface and bottom interactions. Modes whose phase velocity is slower than the speed of sound at the bottom of the lower duct are ducted. Once the ducted modes are identified, their group velocities are used to determine when that mode arrives at a given receiver. The modal analysis results are presented in section 4.1.

\subsection{Signal Processing}

\subsubsection{Active Sonar}

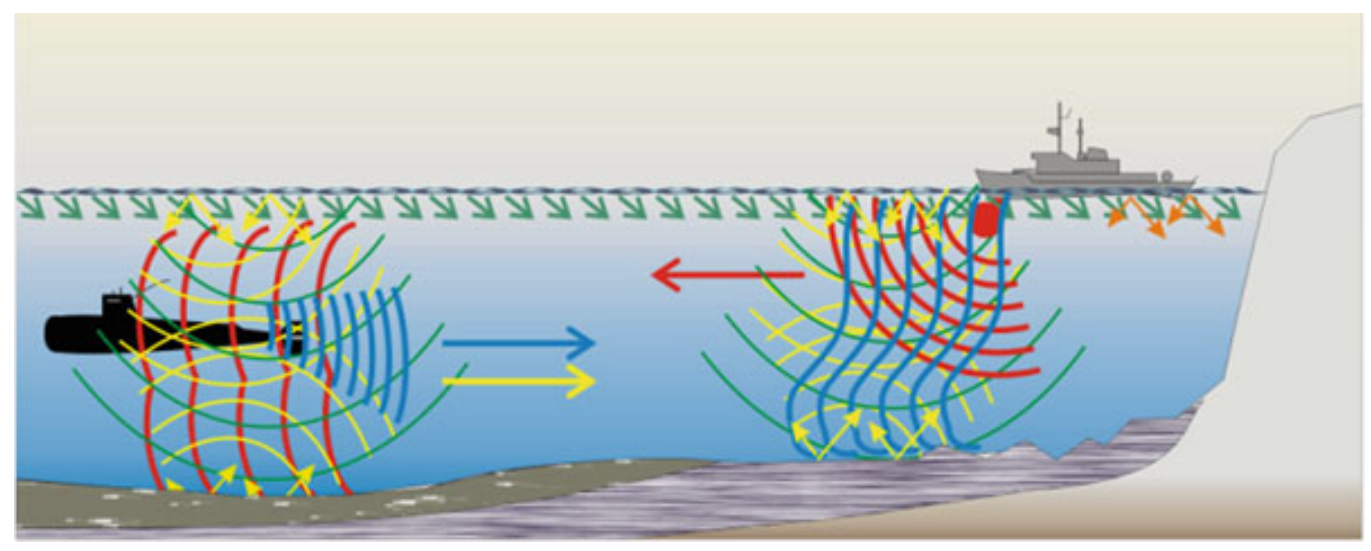

Figure 3.1: An illustration of active sonar from [1].

The focus of this thesis is the impact of the Beaufort Lens on the active acoustic transmissions in the Arctic. The two major applications of active acoustics are sonar and communications. Sonar works identically to radar. A sound waveform is transmitted, reflects off of a target, and returns 
to the transducer. The two-way travel time is then used to calculate target range. Other target characteristics such as speed in the range direction can be determined using Doppler measurements. In passive sonar, no sound pulse is transmitted, and instead, sound emitted from the target is detected. Generally, only the bearing to the target can be immediately determined using array beamforming techniques. More target information can be gradually discerned using fire control systems that build a target solution using geometric relationships. A fire control system is a system that integrates sensor inputs such as sonar, radar, or visual observations to assist in tracking and targeting of a contact.

For either system, the ability to detect a target depends on the signal excess (SE), or the amount of signal (either emitted from the target in passive systems, or reflected from the target in active systems) that can be detected above the ambient noise. The classic active sonar equation is shown in equation 3.24 [1]. SE is the signal excess; SL is source level; TL terms are transmission loss; RL is reverberation loss; $\mathrm{N}$ is noise level; DI is directivity index; DT is detection threshold; All terms are in dB.

$$
S E=S L-T L_{1}+T S-T L_{2}-(R L+N)+D I-D T
$$

Source level, target strength, and directivity index (or array gain) all increase signal excess as they increase. Reverberation loss, noise, transmission loss, and detection threshold all reduce signal excess. This thesis focuses primarily on the effect the Beaufort Lens has on the transmission loss, and noise. Additionally, all sonar simulated in this thesis is monostatic, or the source is also the receiver, thus $T L_{1}=T L_{2}$ and the terms reduce to $2 T L$ due to the 
principal of reciprocity.

The method used for calculating a relative signal excess in this thesis is shown in equation 3.25 .

$$
S E=\int_{f_{\min }}^{f_{\max }} \frac{S_{r}(f)}{S_{n}(f)} d f
$$

$S_{r}(f)$ and $S_{n}(f)$ are the power spectral densities of the received signal and the ambient noise respectively. The integral is only evaluated over the bandwidth of the transmitted sonar pulse. This is not technically a true signal excess as terms such as detection threshold, and directivity index are ignored, but will be referred to as signal excess in this thesis. The equation for evaluating signal excess over a discrete domain is shown in 3.26. The different $\Delta f$ terms account for different frequency bin sizes, if applicable, for the Fourier transforms of the signal and noise.

$$
S E=\sum_{f_{\min }}^{f_{\max }} \frac{S_{r}[f] \Delta f_{S_{r}}}{S_{n}[f] \Delta f_{S_{n}}}
$$

The received pulse can be written in the time domain as:

$$
y(t)=x(t) * h(t) * h(t) * g(t)
$$

Here $x(t)$ represents the original transmitted signal. The Fourier transform of the received signal is:

$$
Y(f)=X(f) H^{2}(f) G(f)
$$




$$
S_{r}(f)=|Y(f)|^{2}
$$

In this form it is easy to see the two-way transmission loss terms from the classic active sonar equation (3.24) in the two convolutions with the channel transfer function, $h(t)$. Equation 3.29 shows the relationship between the received power spectral density term used in equation 3.25 and the fourier transform of the received signal. Additionally, the directivity index term can be incorporated in the filter impulse response, $g(t)$, if beamforming and array processing techniques are used. Matched filters are used in this thesis as all analysis is performed using only a single hydrophone at a time. Matched filtering is explained in section 3.2.2. Equations 3.27 and 3.28 are both applicable for two-way propagation used when modeling active sonar. The same equations still hold for one way propagation applications such as acoustic communication, but the signal is only convolved with the channel transfer function once which results in the $H(f)$ term being raised to the first power.

The three terms from the classic active sonar equation that are ignored in this work are detection threshold, reverberation loss and target strength. Detection threshold is a relatively arbitrary term that can be easily applied to the analysis presented here by simply re-scaling the results. Target strength, or a scattering function based on different targets, is slightly more challenging and presents classification concerns. Thus, perfect reflection from a point spread target is assumed ( $\mathrm{TS}=1$, re $0 \mathrm{~dB})$. 


\subsubsection{Matched Filtering}

In the presence of additive noise, a matched filter provides the maximum signal-to-noise ration (SNR). In the time domain, applying a matched filter consists of convolving the received signal with a conjugated time reversed version of the original input signal, called the signal template. For this thesis, the acoustic pulse is a real signal and thus the matched filter is simply the time reversed acoustic pulse. $x(t)$ represents the time domain input signal; $X(f)$ represents the frequency domain input signal; $y(t)$ and $Y(f)$ represent the received signal and its Fourier transform; and $g(t)$ and $G(f)$ represent the time and frequency domain representations of the matched filter.

The input signal used is the OASES pulse six which is a Hanning windowed sinusoid with frequency centered at the selected center frequency of the pulse [10] and is defined in equation 3.30.

$$
x[n]=\sin \left[2 \pi f_{0} n\right] * \sin ^{2}\left[\frac{\pi n}{N-1}\right]
$$

$f_{0}$ is the center frequency of the source pulse (300 or 3500 in this thesis); $n$ is the discrete time index; and $N$ is the length of the Hanning window which is determined by the bandwidth of the source pulse. This pulse was selected because its bandwidth is easily limited to the desired bandwidth with the Hanning window. The two pulses used in this thesis are a $300 \pm 100 \mathrm{~Hz}$ and $3500 \pm 500 \mathrm{~Hz}$. The lower frequency pulse falls in the frequency band of some long range communication and active sonar applications. The higher frequency band is commonly used for underwater acoustic communication 
and navigation. The two pulses are shown in figures 3.2 and 3.3 and will be referred to as the $300 \mathrm{~Hz}$ pulse and the $3500 \mathrm{~Hz}$ pulse in the following sections. The pulse characteristics are summarized in the table below.

\begin{tabular}{|c|c|c|c|c|}
\hline$f_{0}(\mathrm{~Hz})$ & $f_{\min }(\mathrm{Hz})$ & $f_{\max }(\mathrm{Hz})$ & Bandwidth $(\mathrm{Hz})$ & Power $\left(\mathrm{dB}\right.$ re $\left.\left.1 \mu P a^{2}\right)\right)$ \\
\hline 300 & 200 & 400 & 200 & 193 \\
\hline 3500 & 3000 & 4000 & 1000 & 193 \\
\hline
\end{tabular}
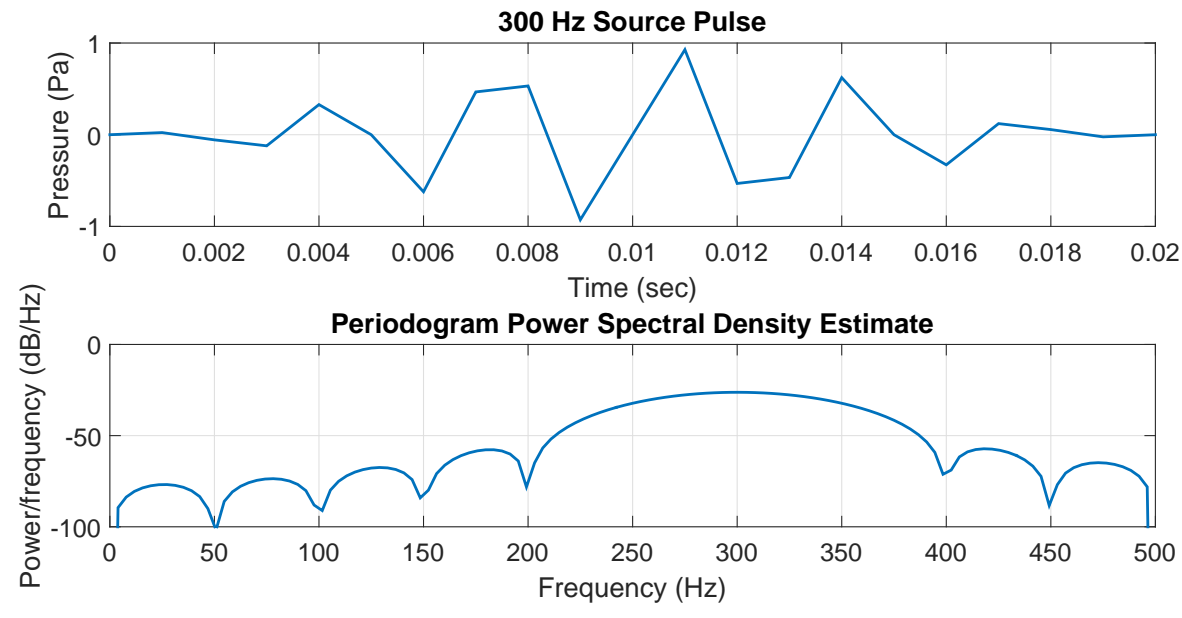

Figure 3.2: The $300 \pm 100 \mathrm{~Hz}$ source pulse.

In addition to matched filtering, most active sonar systems perform a quadrature demodulation to separate the transmitted signal from the complex envelope. This produces a much cleaner return to display to the operator. A simple block diagram of the active sonar system that is modeled in this thesis is shown in figure 3.4. 


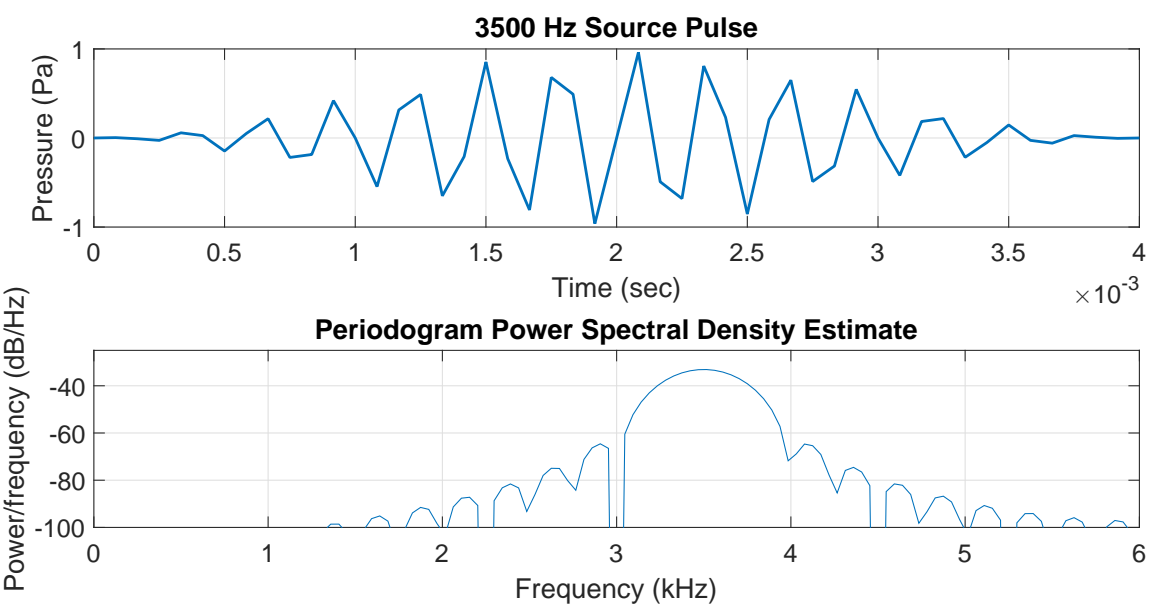

Figure 3.3: The $3500 \pm 500 \mathrm{~Hz}$ source pulse. It is important to note the difference in time scale between the $300 \mathrm{~Hz}$ pulse and the $3500 \mathrm{~Hz}$ pulse.

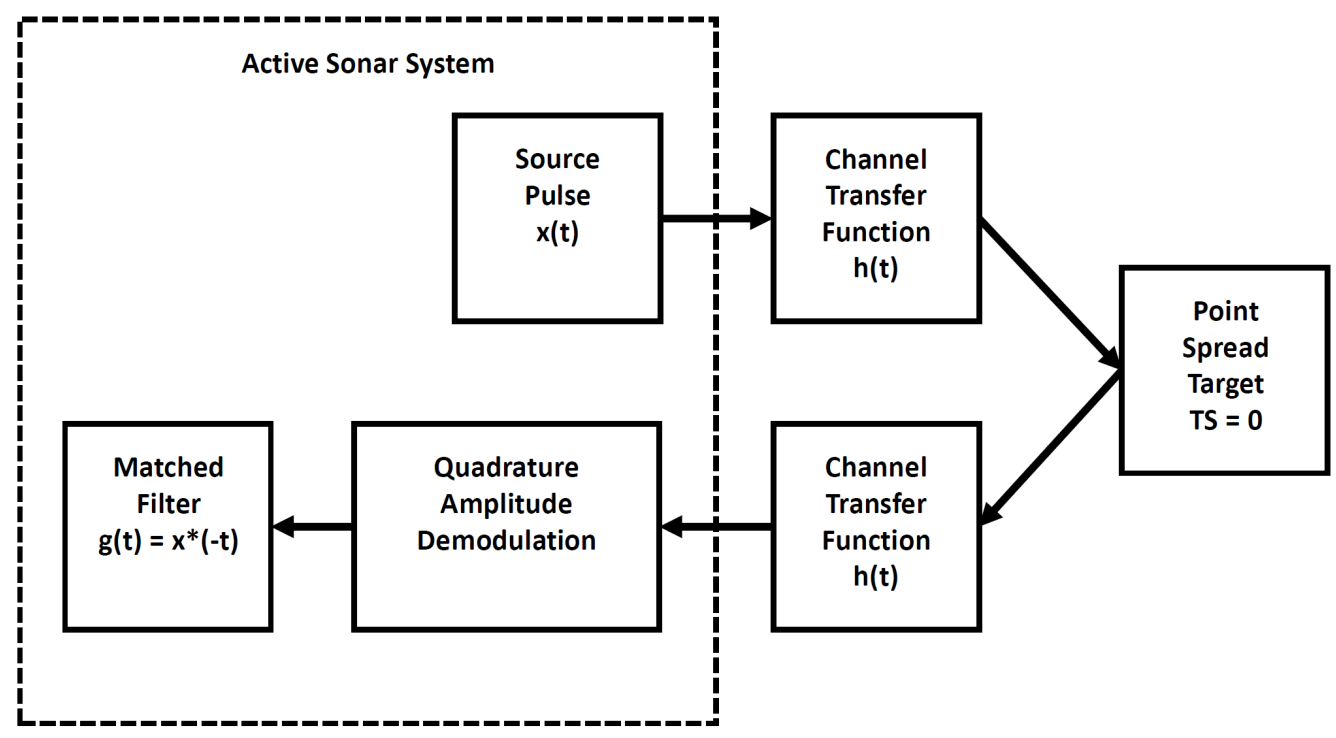

Figure 3.4: A simplified block diagram of the active sonar model used in this thesis. 


\subsection{Channel Capacity}

The channel capacity, in the information theoretic sense, is the highest rate at which data can be transmitted over a channel with an arbitrarily low probability of error. This statistic is useful when designing coding schemes for communications applications, but is used in this work as another method for quantifying the effect of the Beaufort Lens on the Arctic operating environment.

For this thesis the acoustic channel is assumed to be a linear timeinvariant system. This assumption is valid as the SSP does not change over the duration of an acoustic communication signal. Similarly, the SSP is assumed to be range independent. Again this assumption is valid as the SSP is uniform over the region of the tracking range. The channel capacity estimate is obtained by maximizing the integral shown in equation 3.31 [13].

$$
I=\int_{0}^{\infty} \log _{2}\left(1+\frac{|H(f)|^{2} S_{x}(f)}{S_{n}(f)}\right) d f
$$

In equation 3.31, $H(f)$ is the Fourier transform of the channel transfer function, $S_{x}(f)$ is the power spectral density (PSD) of a Gaussian source, and $S_{n}(f)$ is the PSD of the noise. All real sources are limited in their average power output $(\mathrm{P})$ which is included in the maximization as the constraint shown in equation 3.32 [13].

$$
P=\int_{0}^{\infty} S_{x}(f) d f
$$

Using Lagrange multipliers, or the "water-filling" method common in infor- 
mation theory [8], the channel capacity is maximized using equation 3.33. The $L$ term is selected such that the power constraint in equation 3.32 is satisfied.

$$
S_{x}(f)=\max \left(L-\frac{S_{n}(f)}{|H(f)|^{2}}, 0\right)
$$

In applications, the sources are not only power limited, but also band limited. The equations above still hold, but the limits of integration are constrained to the frequency band of the source.

OASES is used to calculate the channel transfer function, $\mathrm{H}(\mathrm{f})$. The PSD of the noise is estimated using actual measured noise from the TAP Experiment in 1994 [5], ICEX data, and simulated noise from OASN. 


\section{Chapter 4}

\section{Results}

\subsection{Modal Dispersion}

\subsection{1 $300 \mathrm{~Hz}$ Pulse}

In his thesis [3], Howe performed an initial analysis of which modes are trapped in the lower duct of the Beaufort Lens, but he only analyzed a simulated lower duct and assumed both boundaries of the duct were pressure release boundaries. This thesis uses the full water column which allows for a more thorough analysis of which modes are trapped in the surface duct and which are trapped in the lower duct of the Lens. Figure 4.1 shows the mode shapes of the first 20 modes of a $300 \mathrm{~Hz}$ source pulse in the classic Arctic environment. The mode shape figures show the modal amplitude vs. mode number and depth and are useful tools for visualizing which modes are trapped in the lower duct. Any mode with a non-zero amplitude (blue, yellow, or red) near the surface will interact with surface. As would be 
expected for a monotonically increasing SSP, each mode reaches deeper in the water column and all interact with the ice cover.

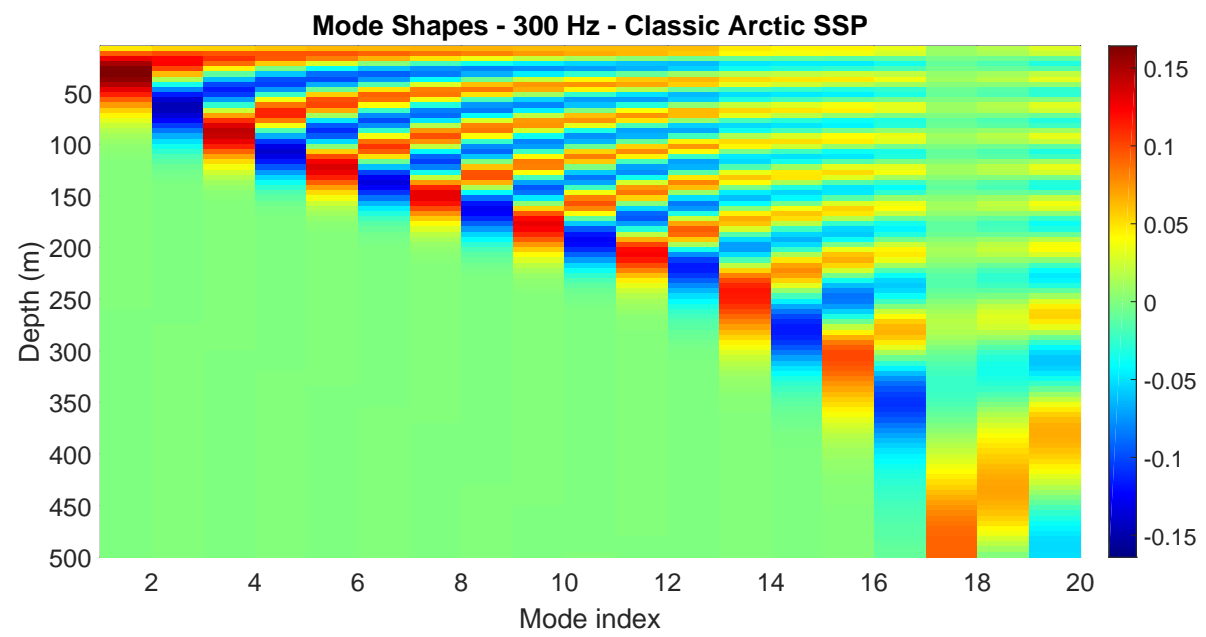

Figure 4.1: Mode shapes of the first 20 modes of a $300 \mathrm{~Hz}$ source pulse in the classic Arctic SSP. The colors reflect the modal amplitude with red being positive and blue being negative. In this case with a typical Arctic SSP, all of the modes interact with the surface and reach deeper and deeper into the water column. The highest order modes will propagate through the entire water depth and interact with both the surface and the bottom.

Figure 4.2 shows the shapes of the first 20 modes with the same source, but in the Beaufort Lens. The first three modes are trapped in the surface duct. Modes 4 through 7 are trapped in the lower duct, with mode 7 starting to leak into the surface duct. Higher order modes all interact with the ice cover, and eventually interact with the bottom as well.

At long ranges, modes 4-7 are the dominant modes. All of the other modes interact with one or both of the boundaries which absorb or scatter the energy. Having isolated the dominant modes, their group and phase speeds are easily determined and plotted vs. frequency in figure 4.3. 


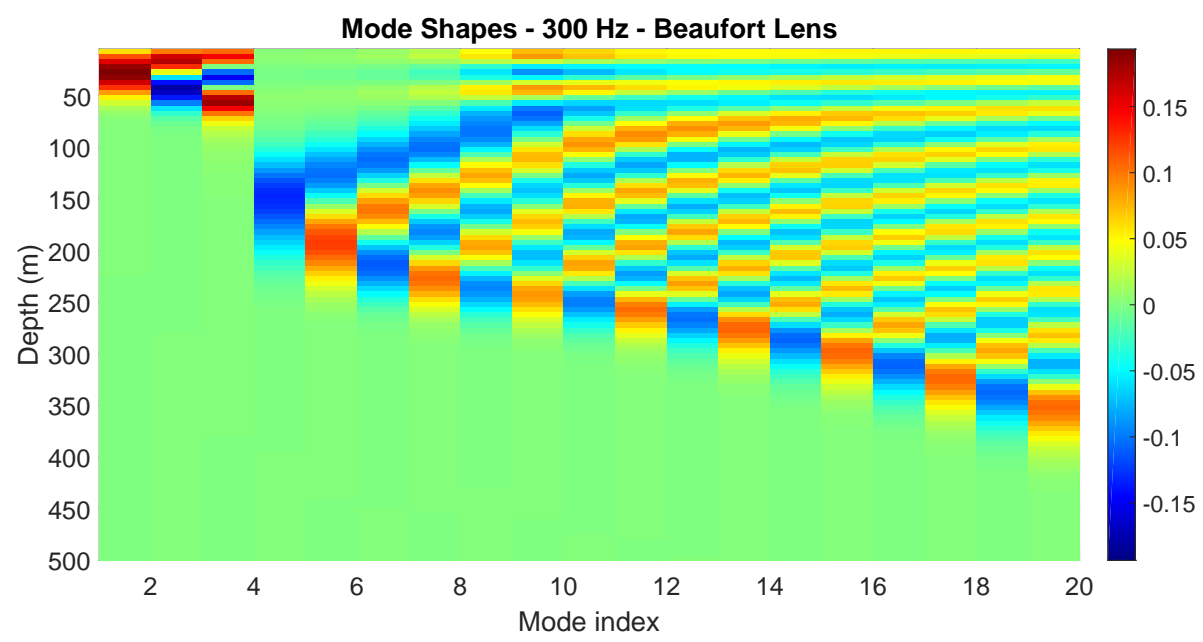

Figure 4.2: Mode shapes of the first 20 modes of a $300 \mathrm{~Hz}$ source pulse in the presence of the Beaufort Lens. In this environment the first three modes are trapped in the surface duct. The 4th through the 7 th mode are ducted in the lower duct with no amplitude at the surface. This means these ducted modes do not undergo any lossy ice or bottom interactions and will propagate to long ranges. 


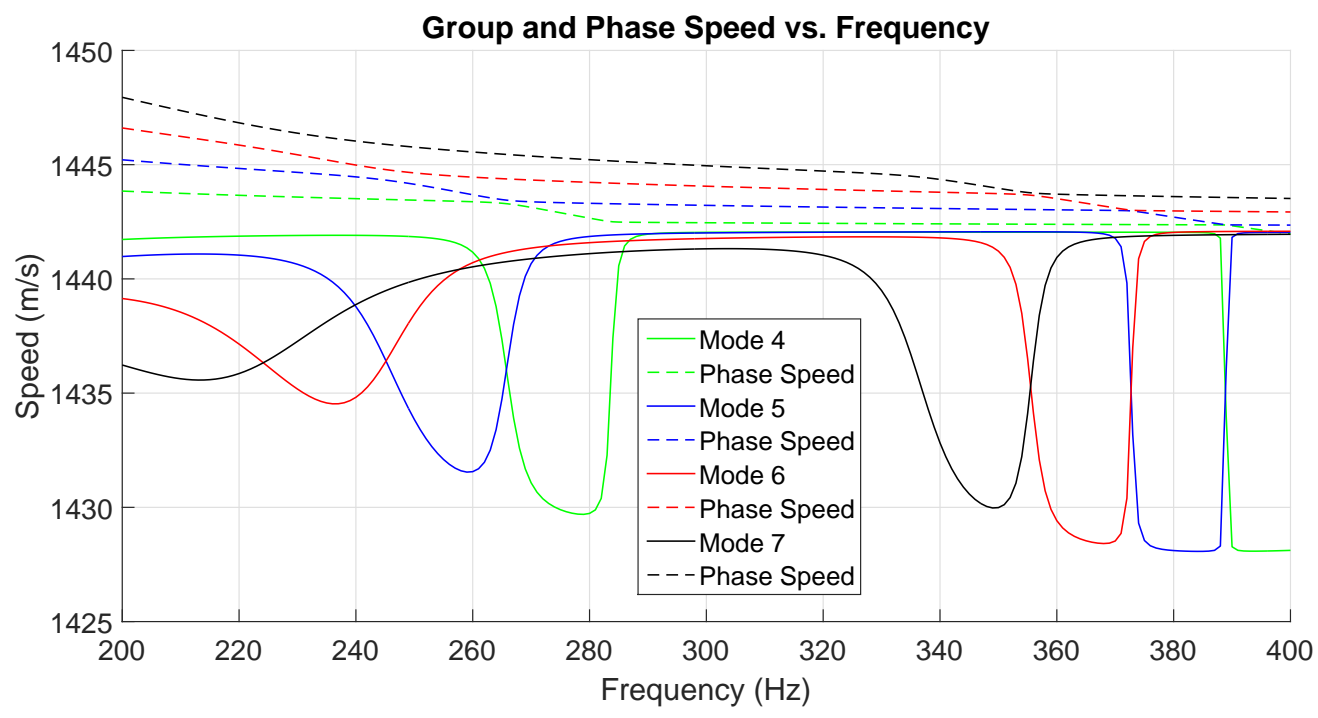

Figure 4.3: Phase and group speeds of modes 4 through 7 vs. frequency in the Beaufort Lens. The group speed is the solid line and the phase speed is the dashed line of the same color. The group speeds cross over each other when the modes "switch" character by occupying the two ducts. The phase speeds also intersect at this same point, but with this frequency sampling, the crossing of the phase speeds is not always visible on this plot. 
The group speed is the speed at which a mode travels through the water. For the $300 \mathrm{~Hz}$ source pulse used, all four of the dominant modes have very similar group speeds with the largest difference being $0.692 \mathrm{~m} / \mathrm{s}$ between modes 5 and 7 . Even at very long ranges, this causes very little modal dispersion. For example, for a two way travel time to a target at a range of 104 kilometers, mode 5 arrives 0.0696 seconds before mode 7 .

The time series plots below show the minimal modal dispersion as well as the significant effect of multi-path propagation and scattering from the ice cover. In the first set of plots the $300 \mathrm{~Hz}$ source is at a depth of 30 meters, in the surface duct. The target is placed at a range of 104 kilometers in the surface duct, and then in the lower duct at a depth of 150 meters. A matched filter is matched to the input signal shown in figure 3.2.
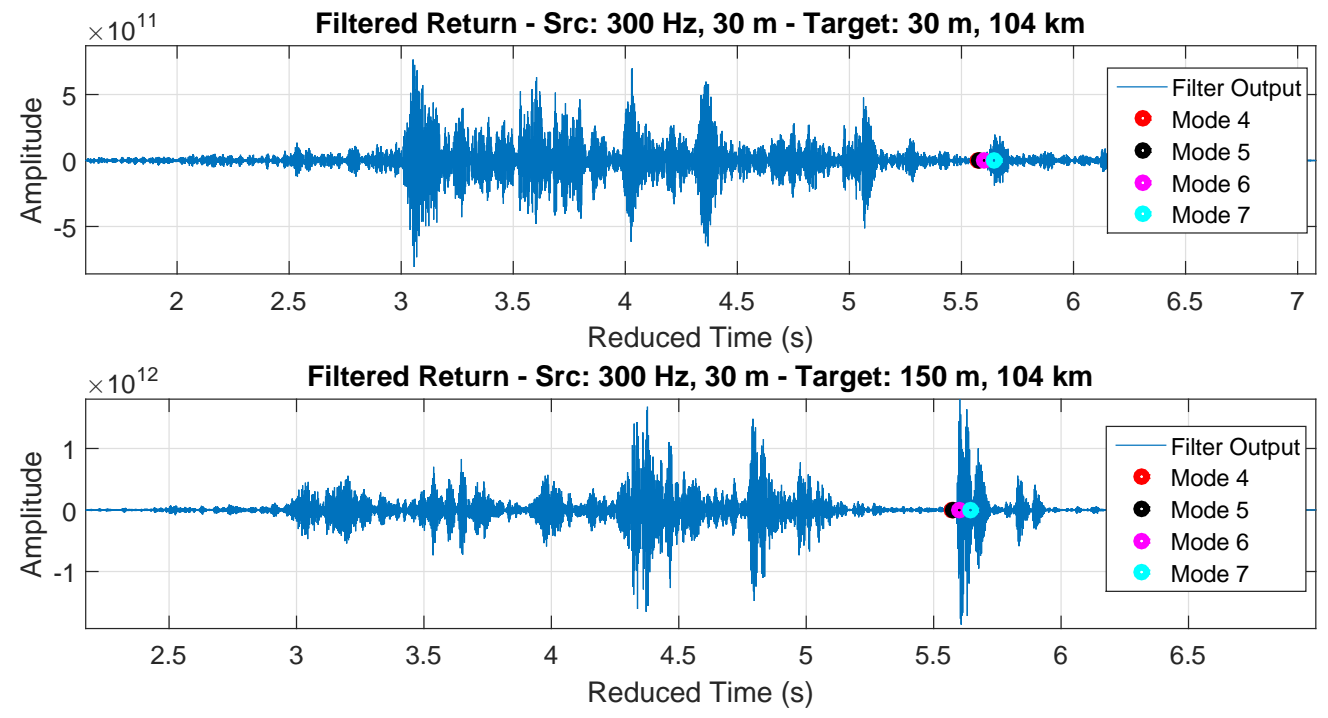

Figure 4.4: Filtered time series for a $300 \mathrm{~Hz}$ source in the surface duct with a target in the surface duct and then in the lower duct with ice cover. 
In both cases the source being in the surface duct results in many multi-path arrivals that are difficult to decipher. When the target is moved into the Beaufort Lens, there is a cleaner pulse returned as a result of the 4 dominant ducted modes being excited, but there is still a lot of clutter that arrives before the refracted/refracted arrival of the ducted waves. The range stacked plots in figure 4.5 highlight how the initially clean pulse spreads and becomes cluttered as the target moves out in range. These time series are plotted vs. the corresponding group speed that is required for each modal arrival. This plotting technique shows that when a target is outside of the duct, the dominant modes are very range dependent and difficult to predict.

Range Stacked Returns for an Active Signal with Two-Way Travel (Reduction Speed = $1500 \mathrm{~m} / \mathrm{s}$ )

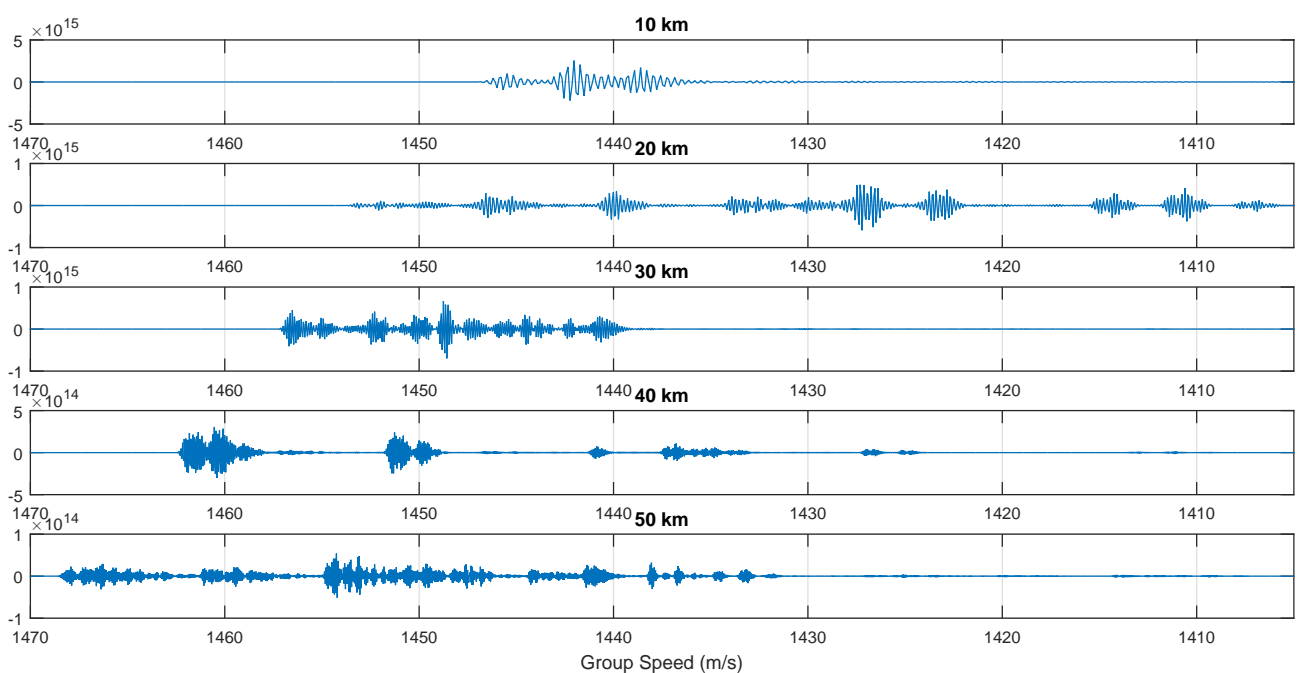

Figure 4.5: Range stacked response for a $300 \mathrm{~Hz}$ source at 150 meters and a target at 30 meters with ice cover in the presence of the Beaufort Lens. The very slow modes that are visible at 20 kilometers are modes from ice interaction and disappear when the ice cover is removed in simulations.

When the source is moved into the lower duct, the results improve 
immensely. With the target in the surface duct, there is still clutter and multi-path arrivals confusing the return (due to the principle of reciprocity this plot is identical to that shown previously where the source was shallow and the target was deep). However, with both the target and the source in the lower duct, there is a very clean return. Modes 4, 5, and 6 are present, and mode 7 does not contribute significantly. This is likely due to the surface interaction and ice loss of mode 7 that was shown in figure 4.2. The response when both the target and the source are in the lower duct shows the new environment's immense potential for active sonar and communication applications.
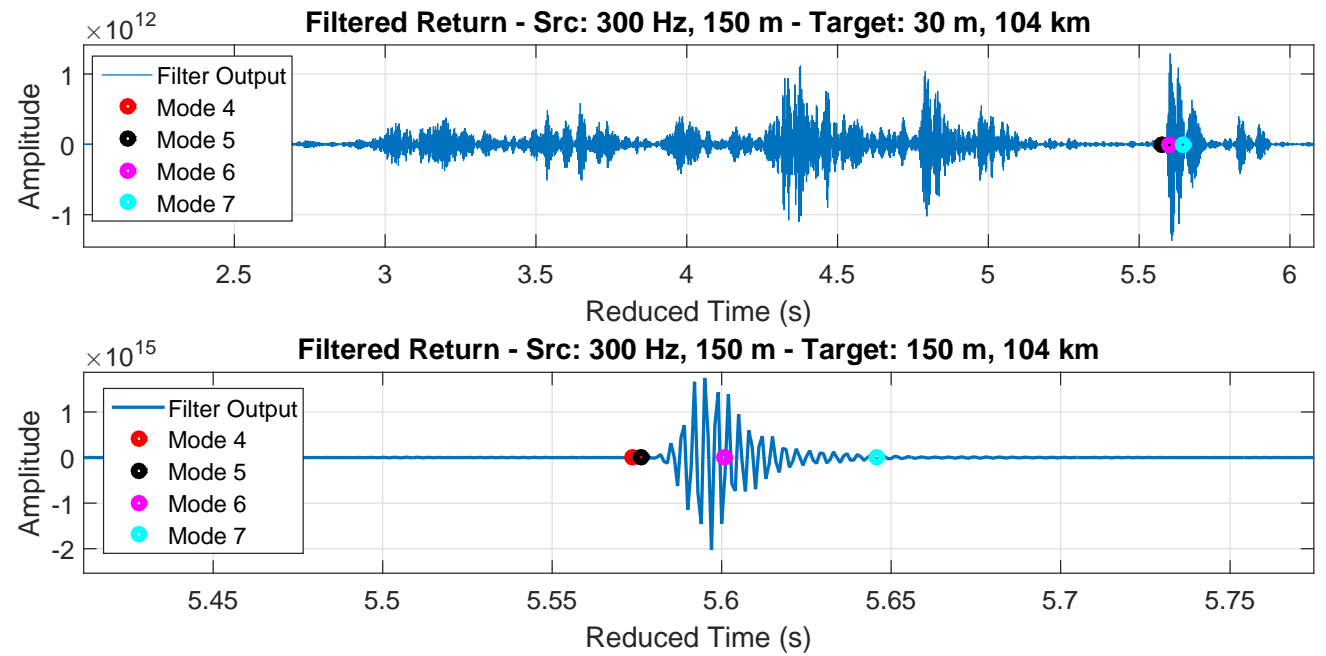

Figure 4.6: Filtered time series for a $300 \mathrm{~Hz}$ source in the lower duct with ice cover in the presence of the Beaufort Lens. The top figure shows the response with the target in the surface duct. The bottom figure shows how clean the response is when the target and the source are in the lower duct. The dots added to the plot show the starting arrival time of the four ducted modes.

The depth stacked responses for a target that is moved in 20 meter 
increments through the duct show that a very clean, three mode (modes 46 specifically) signal is received from about 85 meters to 225 meters. The time series with the target at 265 meters, outside of the duct, illustrates the normal challenges of long range active sonar or communications applications caused by modal dispersion. Figure 4.8 shows the range stacked response for both the source and the target in the duct. The modes that arrive in the duct are consistent and exhibit very little dispersion.

The final plot included in the time series analysis of the Beaufort Lens's impact on a $300 \mathrm{~Hz}$ pulse is a depth stacked plot of the magnitude squared of the filtered signal after it has been demodulated. This plot is shown to replicate the output of a sonar system. Again, in the duct, you can clearly see up to three peaks corresponding to the ducted mode arrivals. On either edge of the duct, faster moving modes start to create cluttered returns. This effect gets worse as you move further away from the duct. 

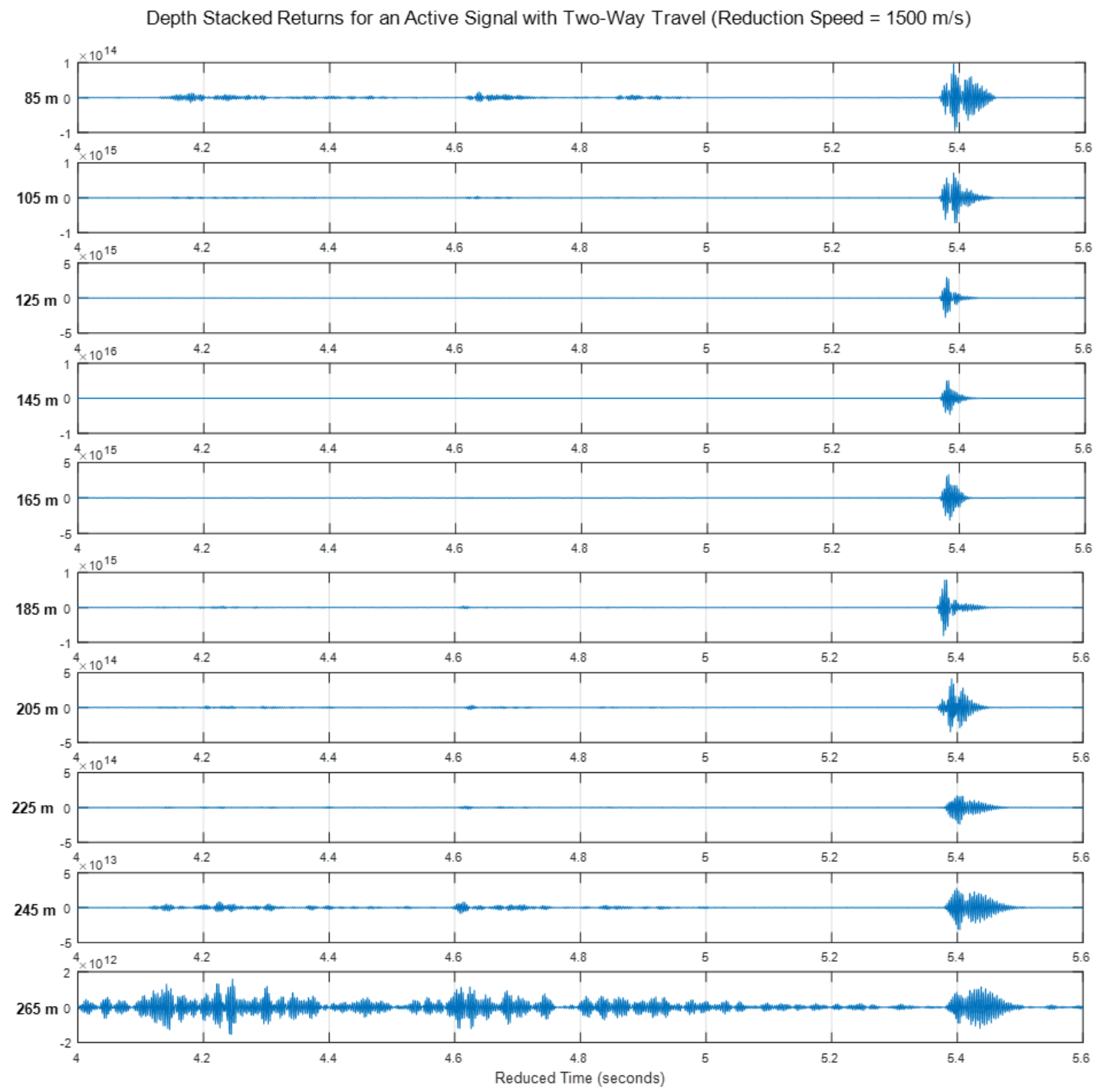

Figure 4.7: Filtered time series for a $300 \mathrm{~Hz}$ source at 150 meters as the target moves from the top of the duct to the bottom of the duct at a range of $100 \mathrm{~km}$ with ice cover. 
Range Stacked Returns for an Active Signal with Two-Way Travel (Reduction Speed = $1500 \mathrm{~m} / \mathrm{s}$ )

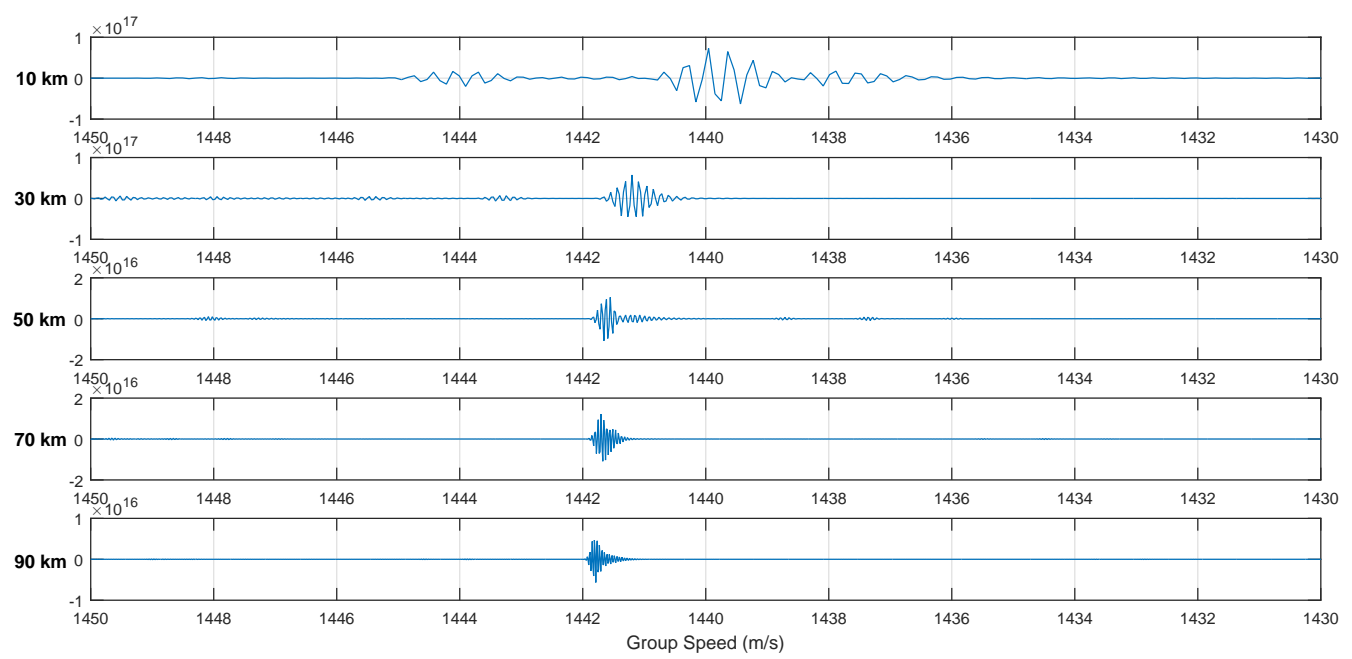

Figure 4.8: Range stacked response for a source and a target at $150 \mathrm{~m}$ in the lower duct. The time series is plotted vs. group speed required for the arrival time which emphasizes how it is the same modes that are dominating the response in the duct at all ranges. It is important to note that by plotting against group speed, the time axis is stretched so the pulses appear to be much more spread out than they are when they actually arrive. 
Depth Stacked Demodulated Returns for an Active Signal with Two-Way Travel (Reduction Speed = $1500 \mathrm{~m} / \mathrm{s}$ )

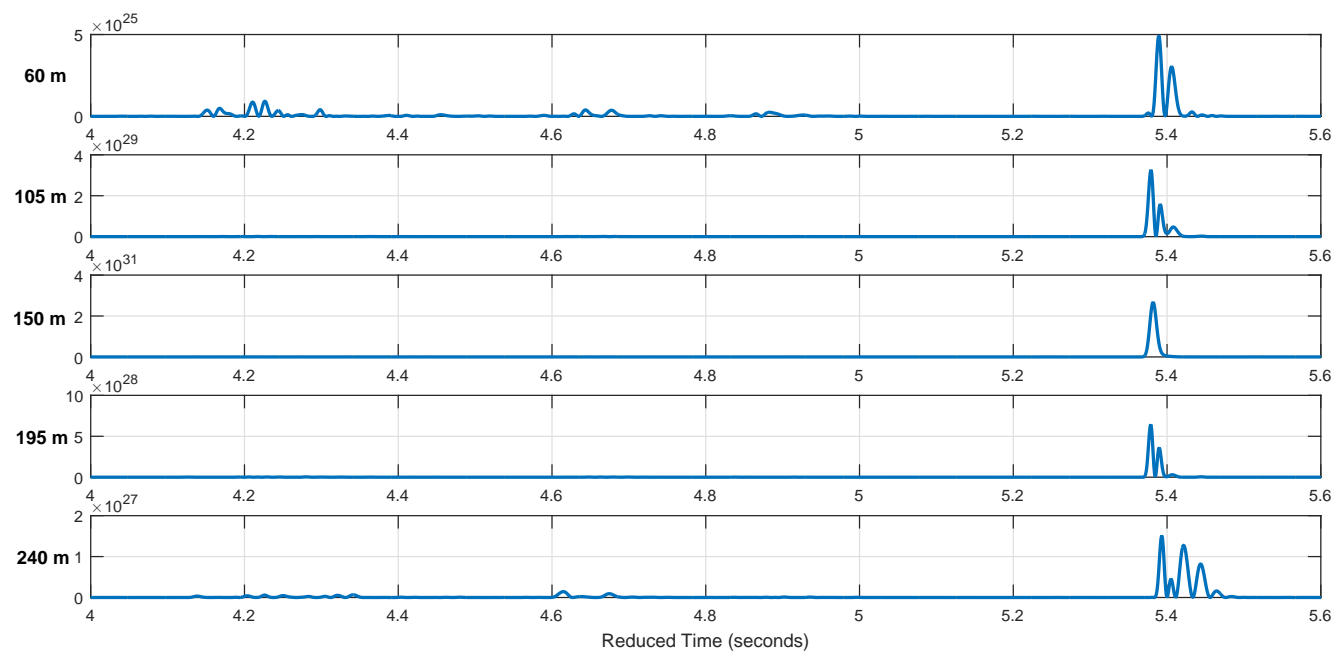

Figure 4.9: Depth stacked demodulated response as the target moves through the duct at 100 kilometers with the source at 150 meters in the Beaufort Lens with ice cover. 


\subsection{2 $3500 \mathrm{~Hz}$ Pulse}

The $3500 \mathrm{~Hz}$ source pulse can fit many more modes in both the surface and the lower duct. The first 33 modes are all trapped in the surface duct. For the most part, modes 33-83 are trapped in the lower duct without any surface or bottom interaction and thus will be the dominant modes at long ranges. Even with 50 ducted modes, the group speed difference between mode 33 and mode 83 is only about $0.5 \mathrm{~m} / \mathrm{s}$ which will result in an arrival time difference of 0.014 seconds for a 60 kilometer travel distance.

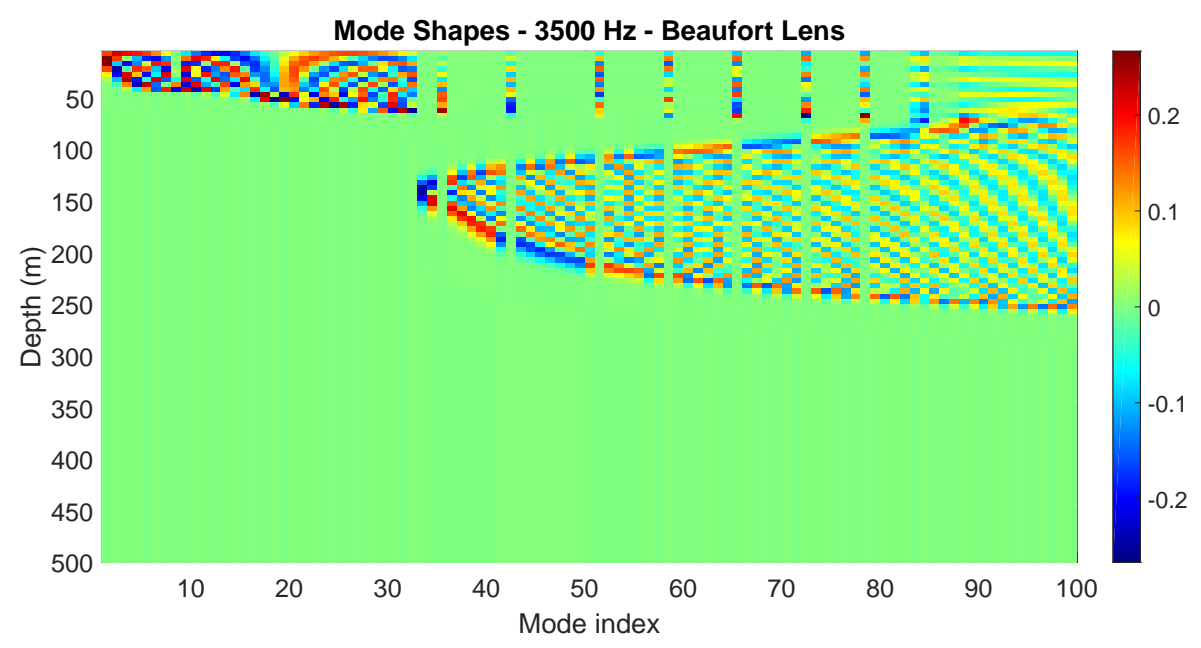

Figure 4.10: Mode shapes of the first 100 modes of a $3500 \mathrm{~Hz}$ source pulse in the presence of the Beaufort Lens.

When examining range stacked arrivals of a $3500 \mathrm{~Hz}$ pulse, more modes are visible as expected. Figure 4.11 shows the range stacked arrivals when both the source and the target are in the duct, at a depth of 150 meters. While there are more modes being ducted than with the $300 \mathrm{~Hz}$ pulse, it is important to note that the group speeds of the ducted modes do not vary significantly. The stretching of the time axis that occurs when plotting the 
received signal vs. group speed is useful for tracking modes, but is misleading in that it enhances dispersion. At ranges out to 30 kilometers, the lack of dispersion of the dominant modes is clearly shown when the time series is plotted vs reduced time instead of group speed as in figure 4.12. Additionally, both the faster and slower modes are modes that interact with the ice cover, but not the bottom. The ice cover is modeled without under-ice roughness for the higher frequency pulse because of limitations of the scattering theory employed by OASES that are explained in section 4.2.2. If roughness is incorporated, these modes would likely scatter and attenuate resulting in a received signal with even less dispersion. Even with the surface interacting modes, the dominance of the ducted modes allows for the easy application of an amplitude threshold that would drop out the cluttered response.

When the target is moved shallow to 30 meters, many more modes are propagating, and therefore the signal becomes much more dispersed. By 30 kilometers, an input signal with a duration of 0.004 seconds is dispersed such that the return is over 0.6 seconds. This is the same effect that is seen with the $300 \mathrm{~Hz}$ pulse and is shown in figure 4.13. Not only is the signal dispersed, but there are no clear dominant modes so an amplitude threshold would not assist in resolving the signal.

The depth stacked response shows that like the $300 \mathrm{~Hz}$ pulse, the 3500 $\mathrm{Hz}$ pulse maintains a consistent modal structure in the Beaufort Lens. The ducted modes dominate the received signal from about 100-210 meters in depth. While there is some dispersion and many more modes still present in the received signal, the ducted modes dominate the response and all arrive around 1.62 seconds in figure 4.14. The variability in the structure of the 


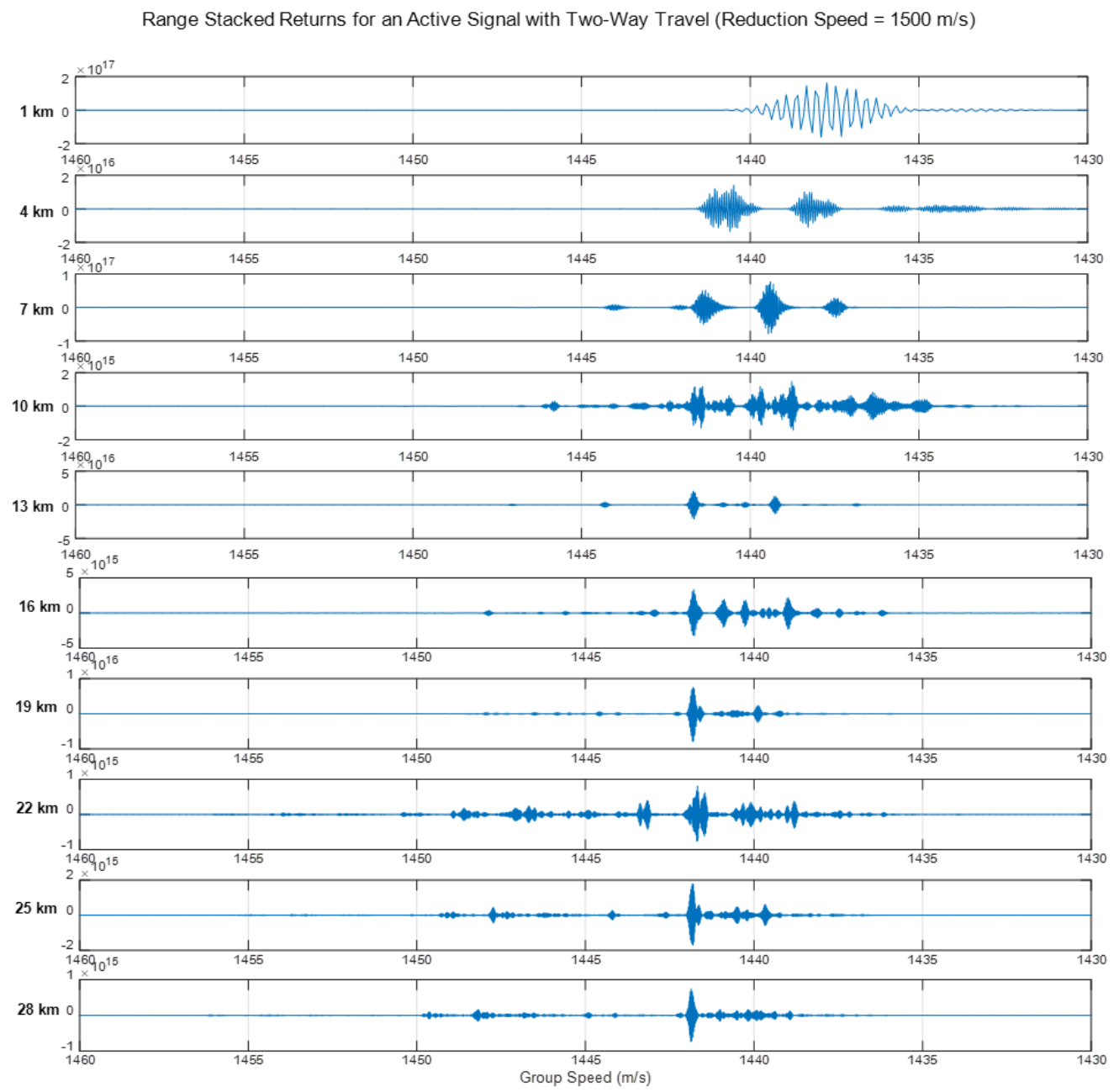

Figure 4.11: Range stacked response for both a $3500 \mathrm{~Hz}$ source and a target in the duct at $150 \mathrm{~m}$. The time series is plotted vs. group speed required for the arrival time which emphasizes how it is the same modes that are dominating the response in the duct at all ranges. 


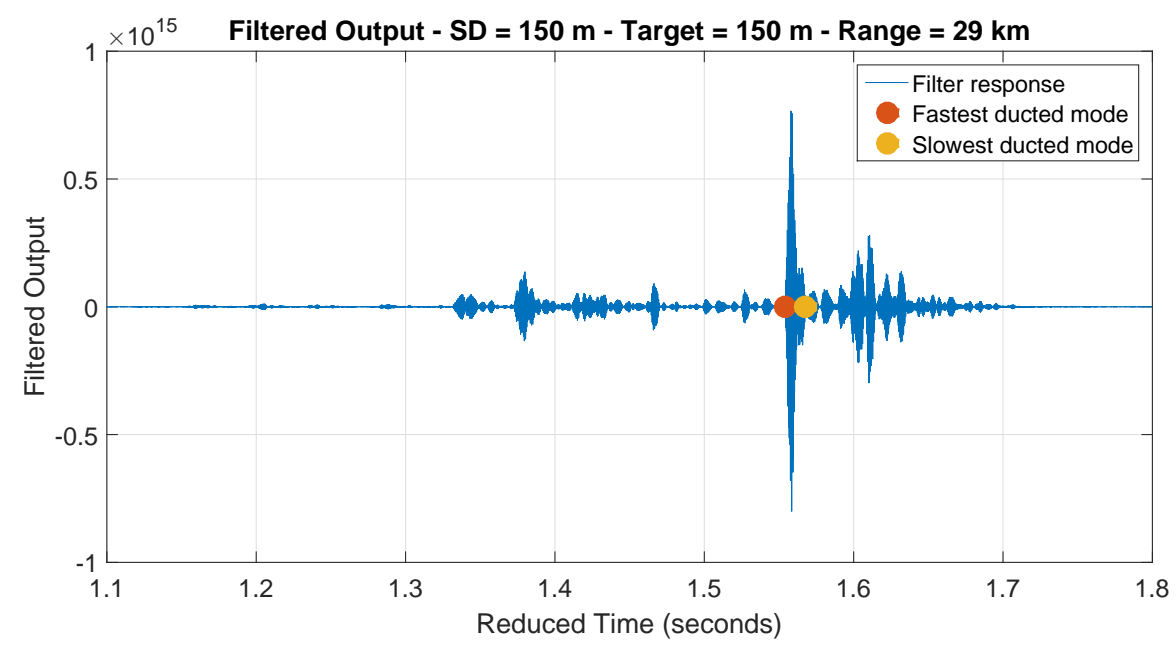

Figure 4.12: Filtered response for active sonar when both the source and target are in the duct. Notice the dominant modes are the ducted modes which arrive between the stars on the plot. The signal that arrives after the ducted modes corresponds to the lower order modes that are trapped in the surface duct. With a more accurate ice model these modes would likely be attenuated or scattered. 


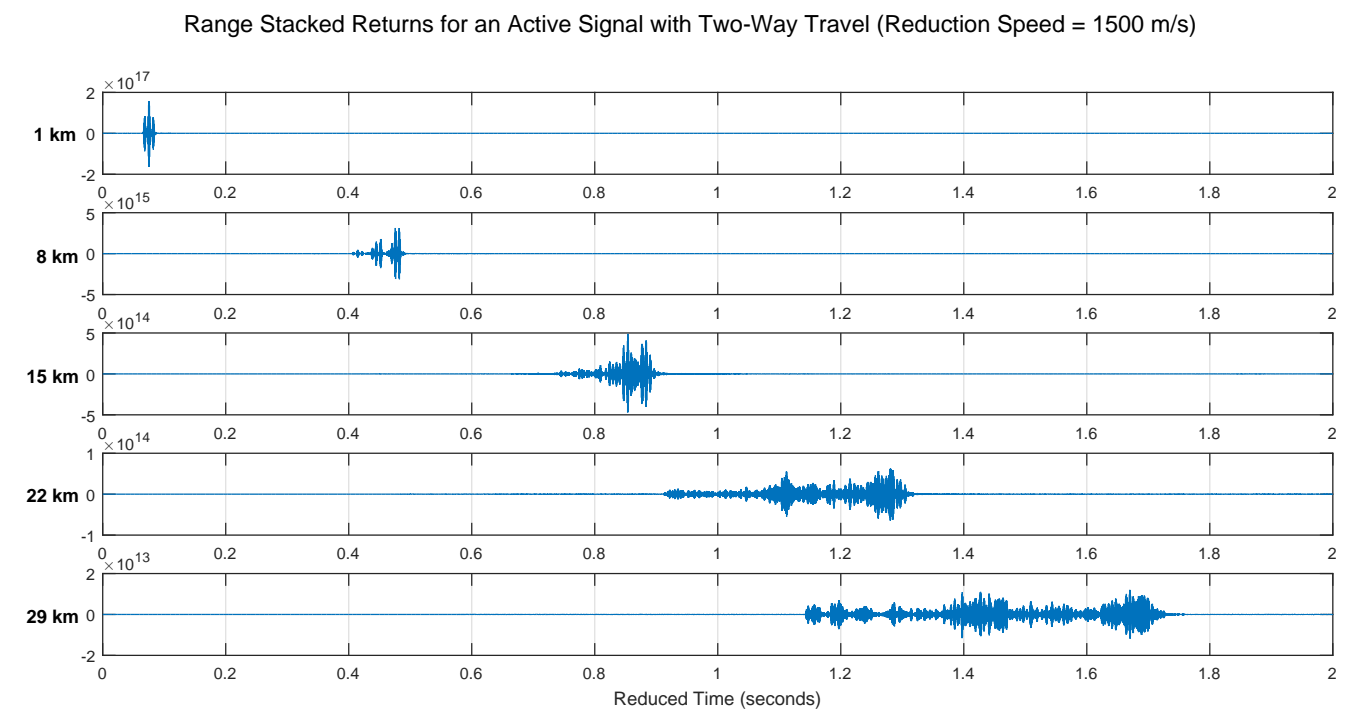

Figure 4.13: Range stacked response for a target in the surface duct at 30 meters with the $3500 \mathrm{~Hz}$ source in the lower duct at 150 meters. Notice how the signal starts relatively clean at 1 kilometer and slowly disperses as the range increases. 
received signal at different depths within the Lens is because of the convergence zone like structure of the refracted/refracted propagation path. Figure 2.5 shows that even within the duct there are areas of higher and lower transmission loss at a given range depending on where in the ray path the target is. The dominant modes are shown more clearly in the demodulated response in figure 4.15. Once again, most of the clutter in the demodulated response comes from the lower order modes that are trapped in the surface duct. These modes would be much weaker if under ice roughness were simulated accurately. 
Depth Stacked Returns for an Active Signal with Two-Way Travel (Reduction Speed $=1500 \mathrm{~m} / \mathrm{s}$ )
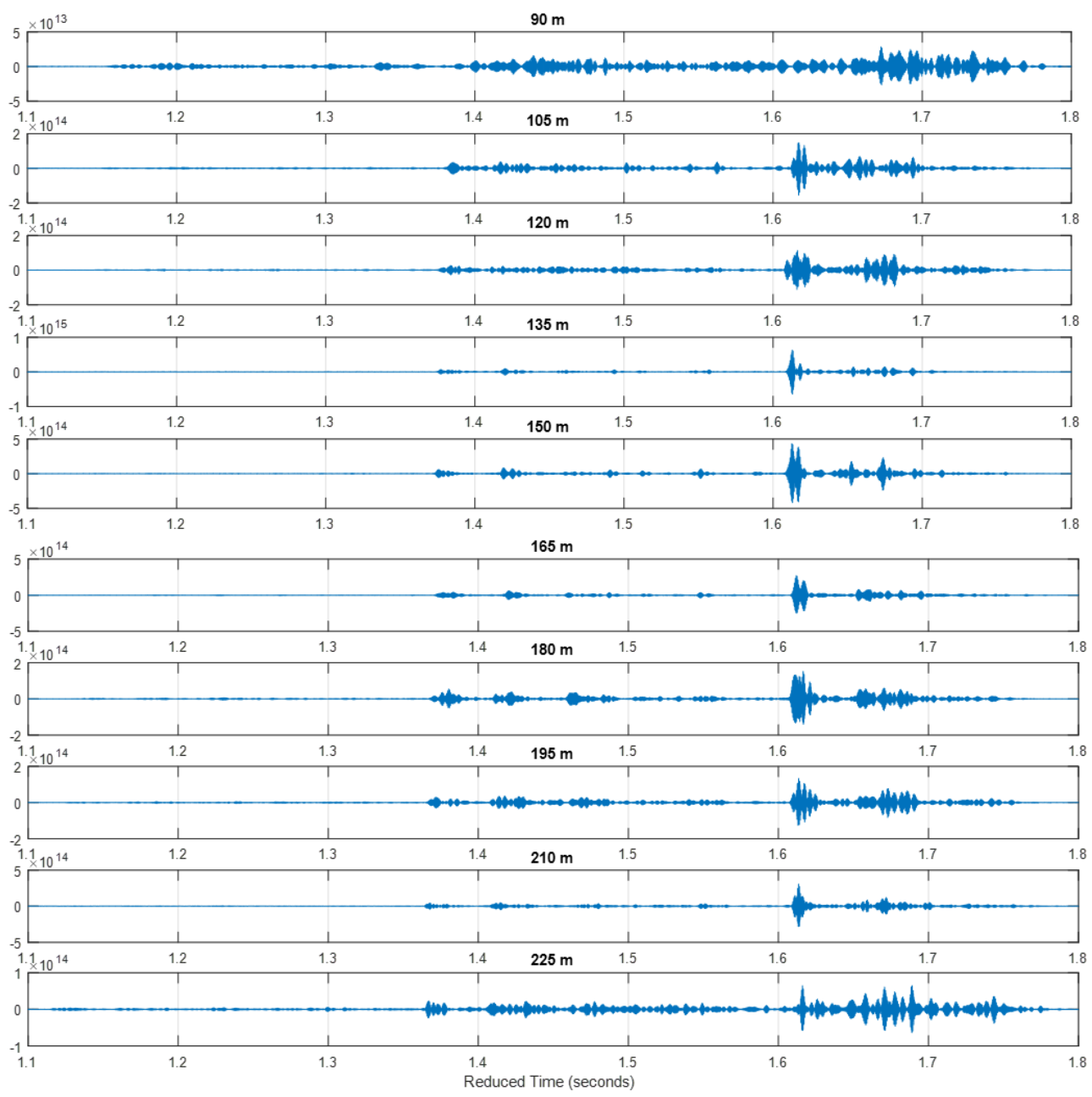

Figure 4.14: Filtered response for a $3500 \mathrm{~Hz}$ pulse for a target at a range of 30 kilometers as it moves vertically through the duct. 
Demodulated Response for a Target at $30 \mathrm{~km}$ (Reduced Speed = $1500 \mathrm{~m} / \mathrm{s}$ )
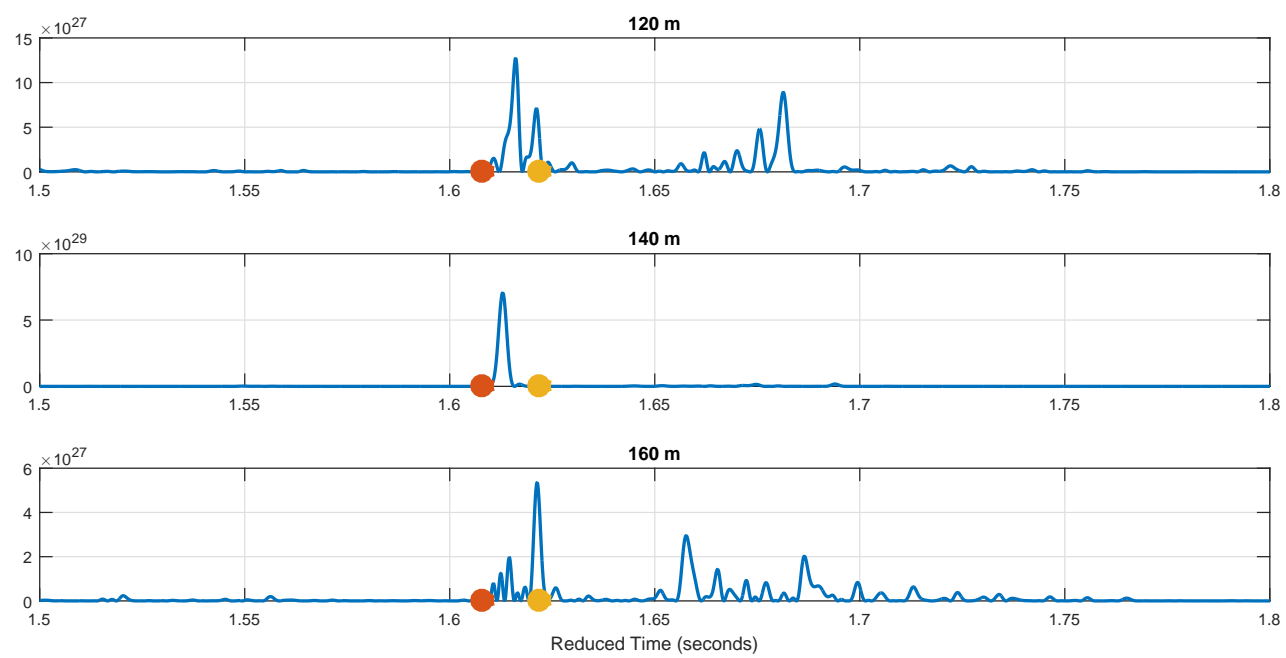

Figure 4.15: Demodulated response for a $3500 \mathrm{~Hz}$ pulse for a target at a range of 30 kilometers as it moves vertically through the duct. The stars on each plot indicate the first and last arrival times of the ducted modes and show that the dominant modes that are those that are trapped entirely in the lower duct. The later returns correspond to lower order modes that are trapped in the surface duct and would be scattered or attenuated if the ice roughness were modeled accurately. 


\subsection{Signal Excess}

\subsection{1 $300 \mathrm{~Hz}$ Pulse}

The $300 \mathrm{~Hz}$ source analyzed in section 4.1 and shown in figure 3.2 is used to determine signal excess. A two-way propagation is calculated to simulate active sonar and the matched filter used in section 4.1 is used to maximize SNR. The included plots show the signal excess at the source for a target at the specified depth and range. Initially, the noise is assumed to be uniform throughout the water column. The data collected from the TAP experiment and shown in figure 2.7 are used. The assumption of depth independent noise is used to highlight the effect of the SSP on the channel transfer function since Arctic noise is very diverse and can change dramatically due to weather and ice conditions. In reality, the noise is not uniform in depth as the environment shapes the noise just as it shapes the signal. A brief analysis of the effect of the Beaufort Lens on the shape of the ambient noise is included at the end of this section. However, there is no analysis of the vertical directionality of the noise which can be exploited to enhance signal excess if a hydrophone array is used. All analyses presented in this thesis use a single hydrophone receiver. Figures 4.16 and 4.17 show the signal excess of a $193 \mathrm{~dB}$ re $1 \mathrm{~m}$ and $1 \mu \mathrm{Pa}$ source at a depth of 30 meters in the classic Arctic and in the presence of the Beaufort Lens respectively. A 193 $\mathrm{dB}$ source is used as it is approximately the same power of the HLF-3 source that was used for the TAP experiment and is a common source power used in the literature. With the exception of figure 4.18, all of the figures shown include the ice cover. 


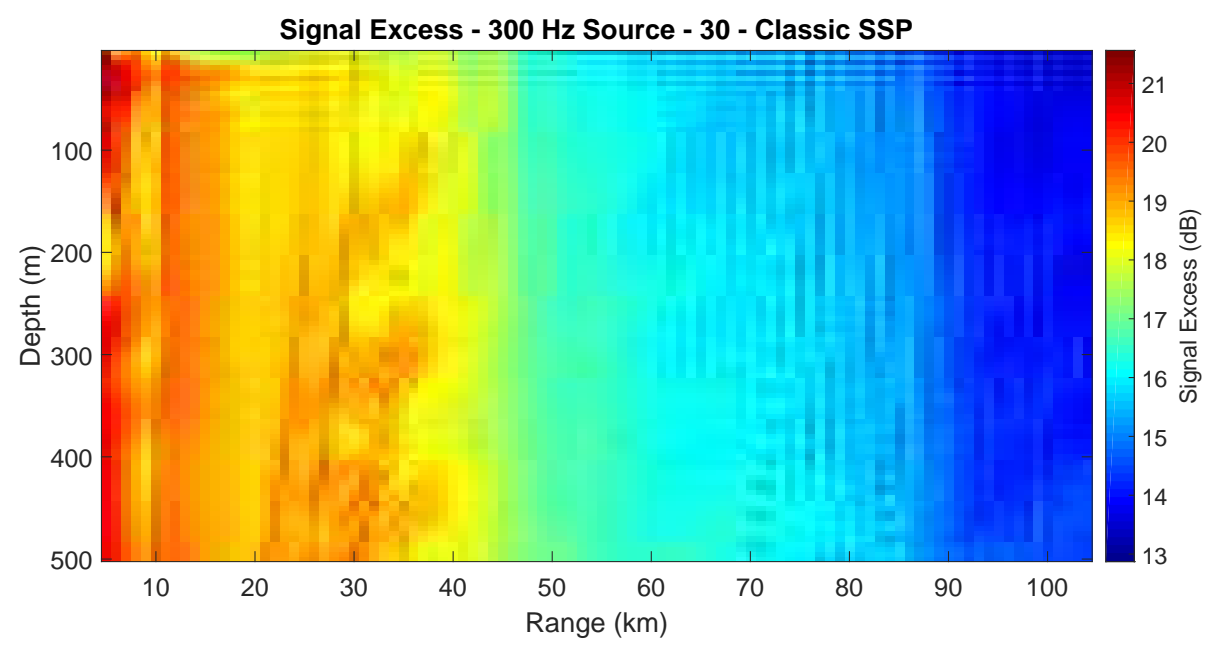

Figure 4.16: Signal excess for a $300 \mathrm{~Hz}$ source at 30 meters in the classic Arctic with ice cover.

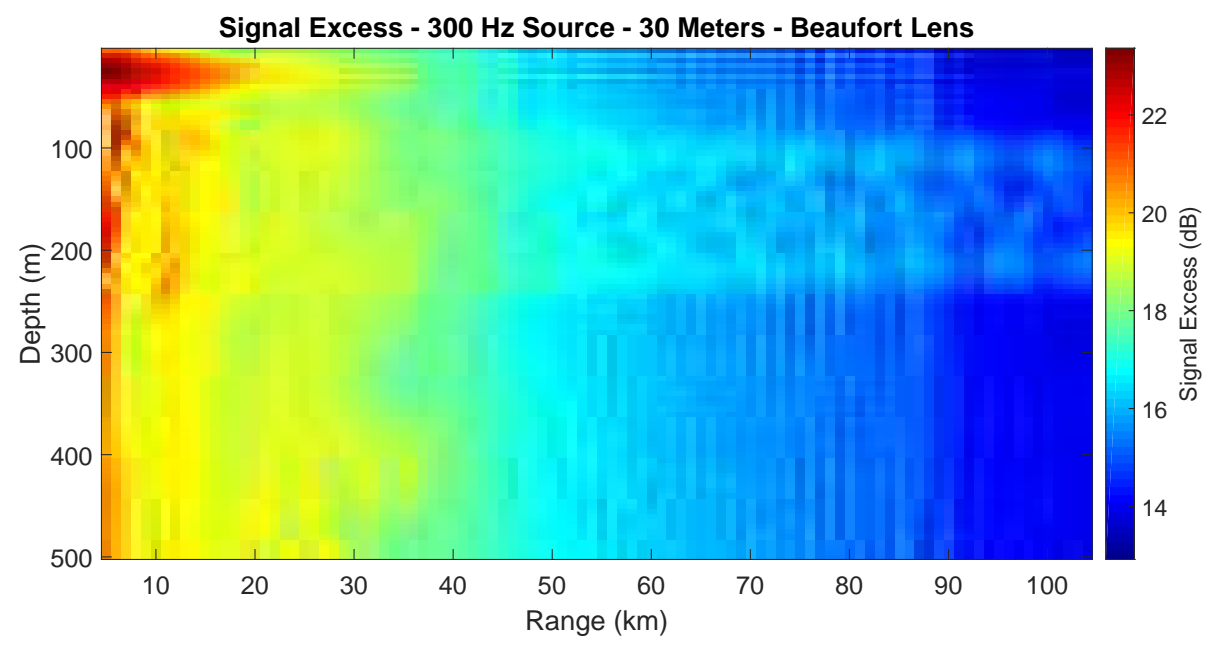

Figure 4.17: Signal excess for a $300 \mathrm{~Hz}$ source at 30 meters in the presence of the Beaufort Lens with ice cover. Even though the source is above the duct, some of the sound is still trapped in the lower duct and the ducting effect is visible. 
In the classic environment, signal excess remains fairly uniform in depth down to 500 meters, and gradually decreases with range. In the presence of the Beaufort Lens, signal excess is more depth dependent as much more energy is trapped in the surface duct when the source is at 30 meters. Figure 4.18 shows the same signal excess calculation as in figure 4.17 , but the ice cover is removed. Without ice, the surface duct propagates the energy to long ranges similarly to the lower duct. This figure is included to highlight the importance of accurately modeling the ice and under-ice roughness.

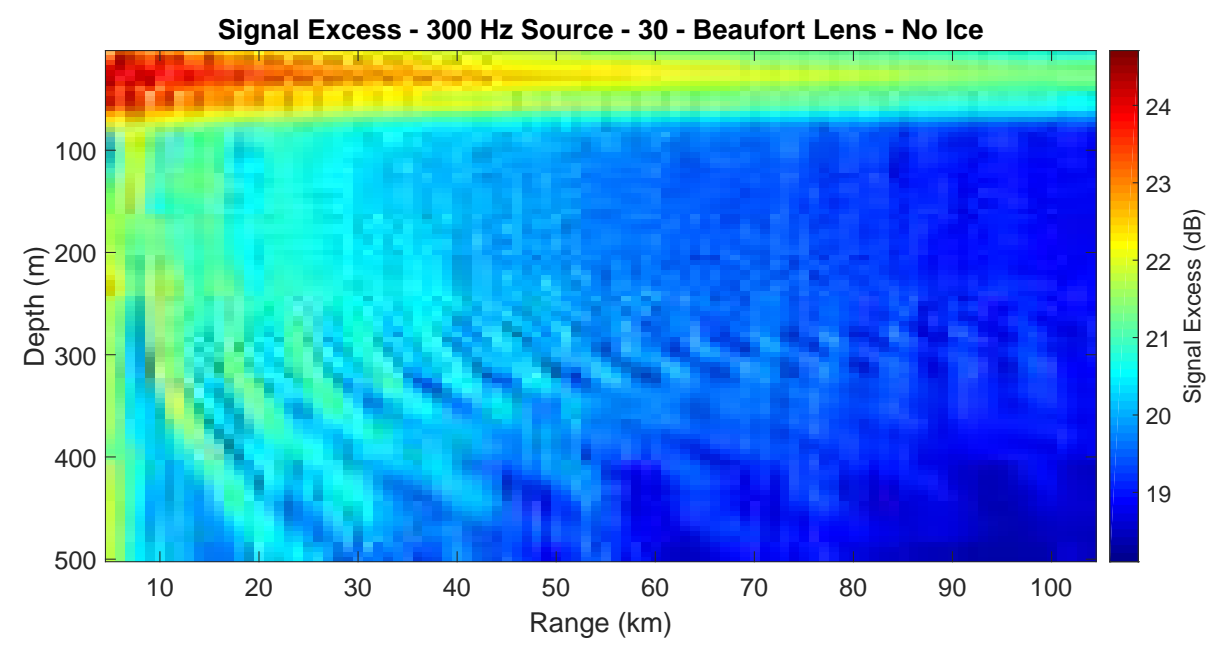

Figure 4.18: Signal excess for a $300 \mathrm{~Hz}$ source at 30 meters in the presence of the Beaufort Lens with no ice cover. Without any ice cover and under-ice roughness, the signal in the surface duct propagates to long ranges.

As shown in section 4.1, the effect of the Beaufort Lens can be used to great benefit when the source is lowered into the duct. Figure 4.19 shows the effect on signal excess when the source is lowered to 150 meters.

When the source is lowered to the center of the lower duct, a high signal excess is maintained for long ranges. To emphasize this effect, figure 


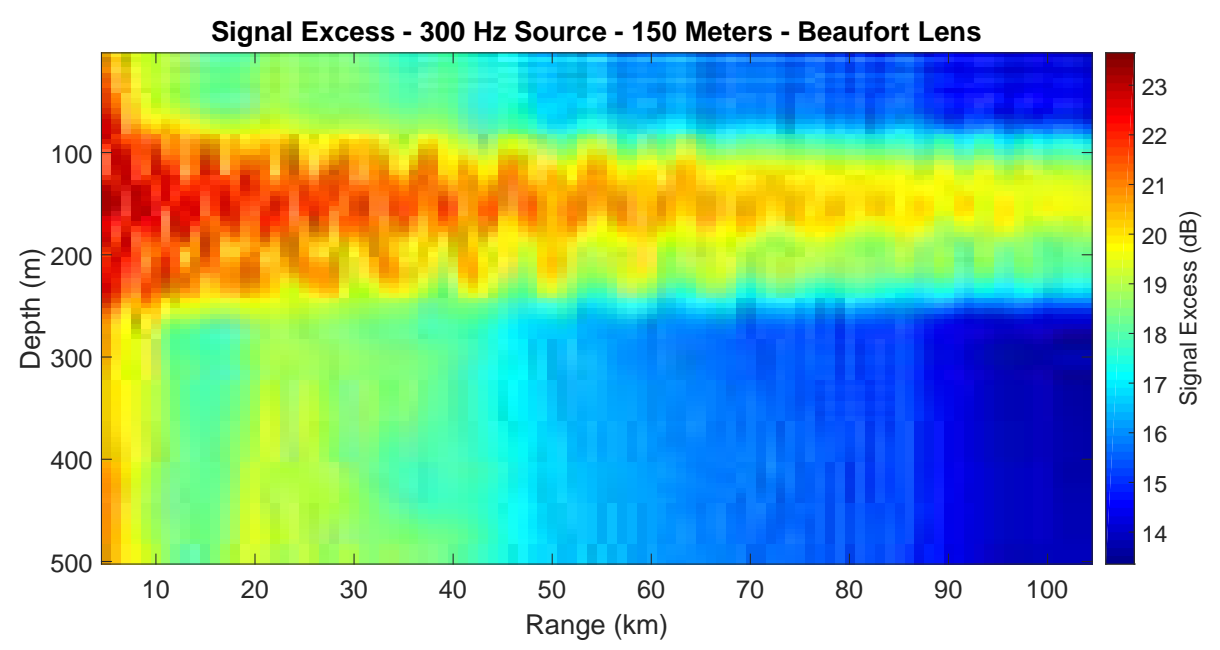

Figure 4.19: Signal excess for a $300 \mathrm{~Hz}$ source at 150 meters in the presence of the Beaufort Lens with ice cover.

4.20 shows the signal excess difference between a shallow and deep source in the Beaufort Lens. Lowering the source from 30 meters to 150 meters creates a $6 \mathrm{~dB}$ gain in signal excess in the Lens for a target at 104 kilometers. The figure also shows a $4 \mathrm{~dB}$ improvement in signal excess at short ranges for a shallow target if the source is kept shallow. While this makes sense, it is likely not as significant as the figure shows. The calculation assumes that any energy in the bandwidth of interest is useful signal. The scattering effect of the ice does not necessarily attenuate the energy at short ranges, but creates significant clutter so a lot of the returns are not useful signal.

So far, everything shown has been for active sonar applications with a two way travel time. For acoustic communications, the signal need only to propagate to the target. Figure 4.21 shows the signal excess for a one way travel time. The structure of the contour plot is the same as for active sonar, but, as would be expected, the signal excess increases by about $6 \mathrm{~dB}$. 


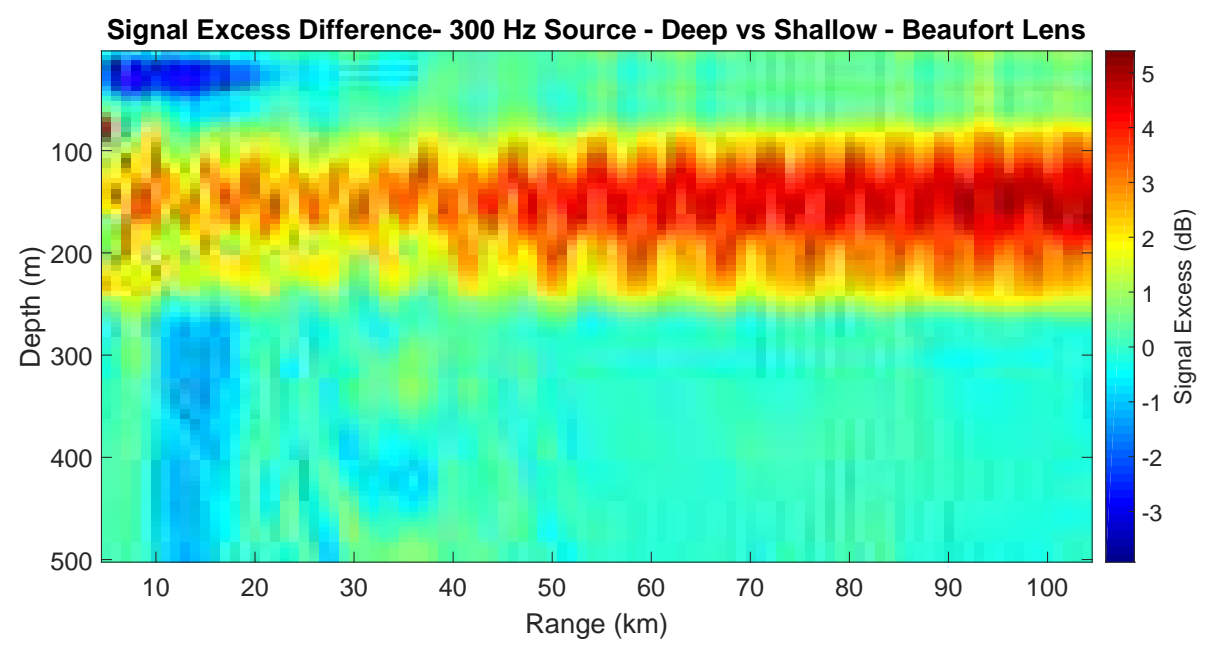

Figure 4.20: Signal excess difference for a $300 \mathrm{~Hz}$ source at 150 meters and at 30 meters in the new Arctic. Warmer colors indicate an enhancement of signal excess by lowering the source into the lower duct.

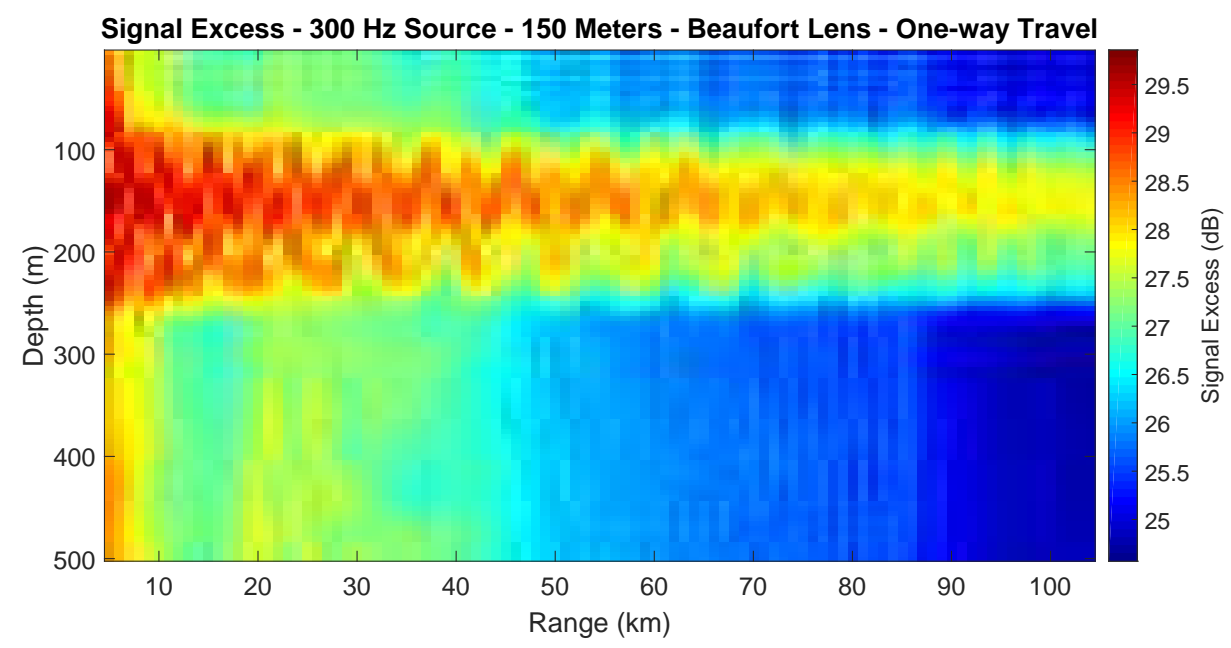

Figure 4.21: Signal excess for a $300 \mathrm{~Hz}$ source at 150 meters in the new Arctic with only one-way propagation. 
The assumption of depth independent noise is not very accurate as the environment that so dramatically affects the propagation of a signal has similar effects on the ambient noise. Most ambient noise in the Arctic is generated at the surface and thus noise power decreases with depth. Figure 4.22 shows the depth dependence of noise in the classic Arctic environment as well as in the new Arctic and was generated using OASN.

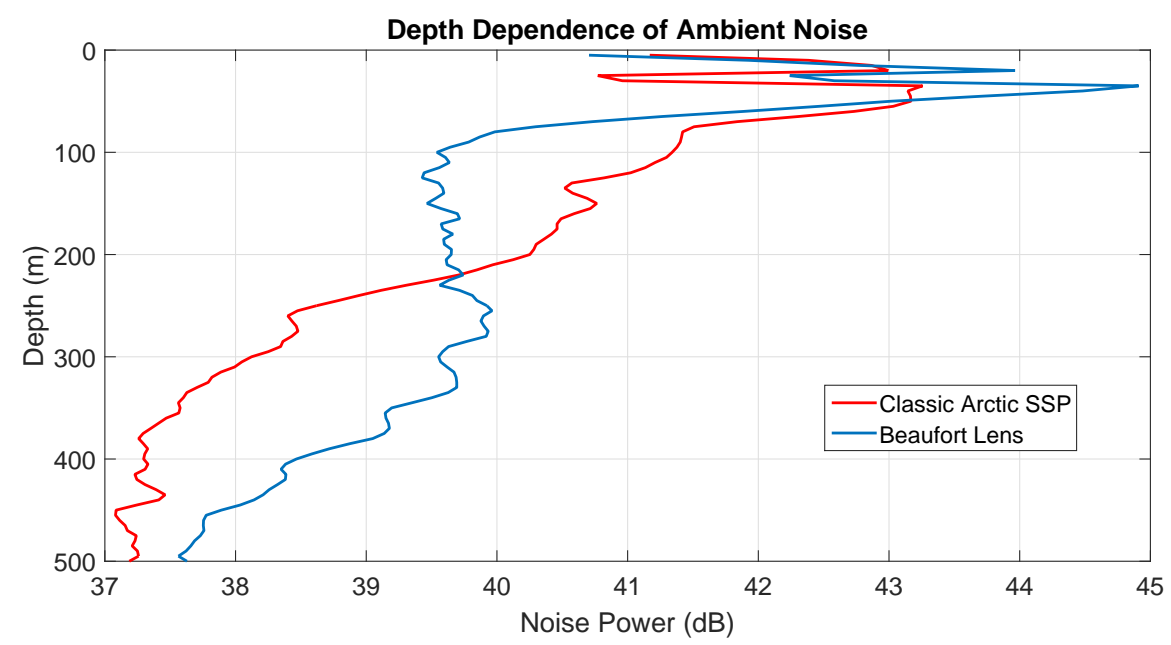

Figure 4.22: Noise power vs. depth for a $300 \mathrm{~Hz}$ source as predicted by OASN.

In the new Arctic, the surface duct is more pronounced so noise is more effectively ducted near the surface, increasing noise power in the surface duct by about $2 \mathrm{~dB}$ compared to the classic SSP. In the lower duct, the noise power is reduced by about $1 \mathrm{~dB}$ so not only does the duct serve as a waveguide for the signal, it provides an environment with lower ambient noise. Figure 4.23 shows the signal excess for a source in the lower duct with a depth dependent noise profile. As expected, the signal excess is even better in the duct than it was with depth independent noise. Not only is the signal excess enhanced 
inside the Beaufort Lens, but it is lower at depths outside of the lens when the depth structured noise is modeled.

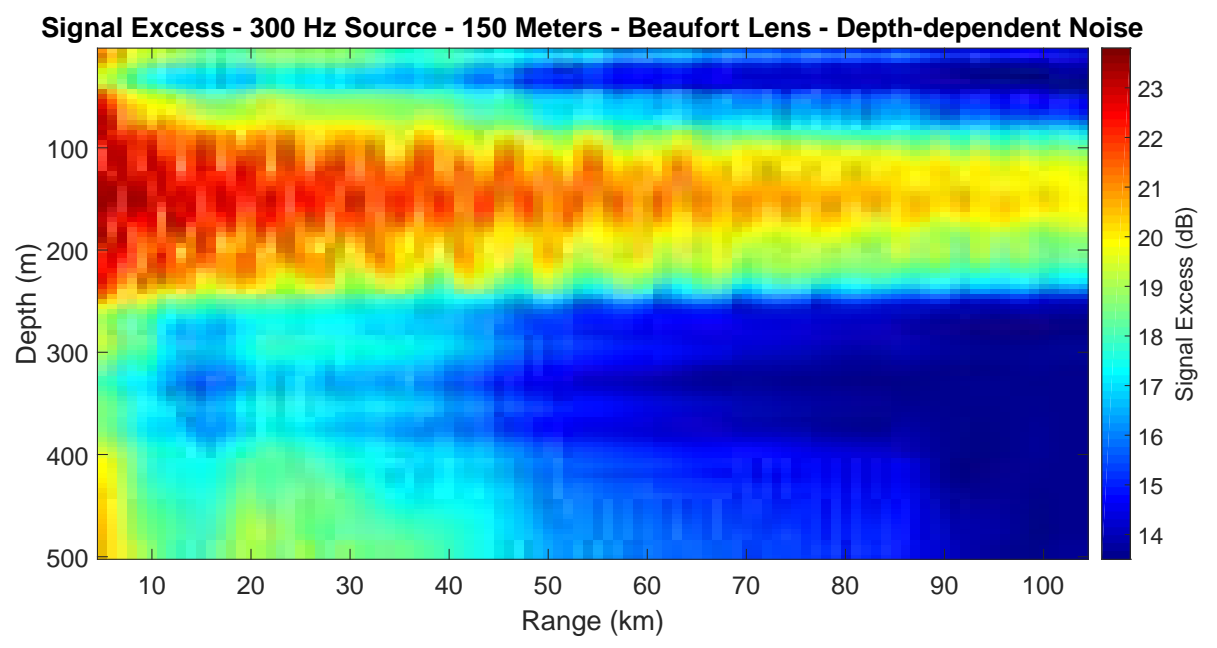

Figure 4.23: Signal excess for a $300 \mathrm{~Hz}$ source at 150 meters in the new Arctic with depth-dependent noise. 


\subsection{2 $3500 \mathrm{~Hz}$ Pulse}

The structure of the signal excess plots looks similar between the 300 $\mathrm{Hz}$ pulse and the $3500 \mathrm{~Hz}$ pulse. One major difference between the plots is that the $3500 \mathrm{~Hz}$ pulse is modeled with a smooth ice cover while the 300 $\mathrm{Hz}$ pulse models incorporate under-ice roughness. The under-ice roughness is important as it adds realistic loss and scattering to the model, but the scattering theory employed by OASES only is valid when the ice roughness correlation length is small compared to the wavelength. For the $3500 \mathrm{~Hz}$

pulse, the much smaller wavelength invalidates the scattering theory so the roughness parameters need to be adjusted. Accurate parameters for this frequency band are not known. Figures 4.24 and 4.25 shows the signal excess for a shallow source in the classic Arctic environment and in the presence of the Beaufort Lens respectively. These figures are only plotted to a range of 30 kilometers because of the computational cost of modeling such a high frequency and large bandwidth. Additionally the modal dispersion and attenuation of the higher frequency preclude most useful applications beyond 30 kilometers.

One important characteristic of the double ducted environment that is highlighted in figure 4.25 is the heavy shadow zone between about 75 and 250 meters at ranges up to 6 kilometers. This shadow zone is what made acoustic communications and tracking of the AUV and submarines in ICEX so challenging and had not been seen in prior exercises.

Lowering the source into the duct has the same effect as it does for the lower frequency pulse. Again, the smooth ice model creates an artificially high signal excess in the surface duct. In reality, the plot should look even 


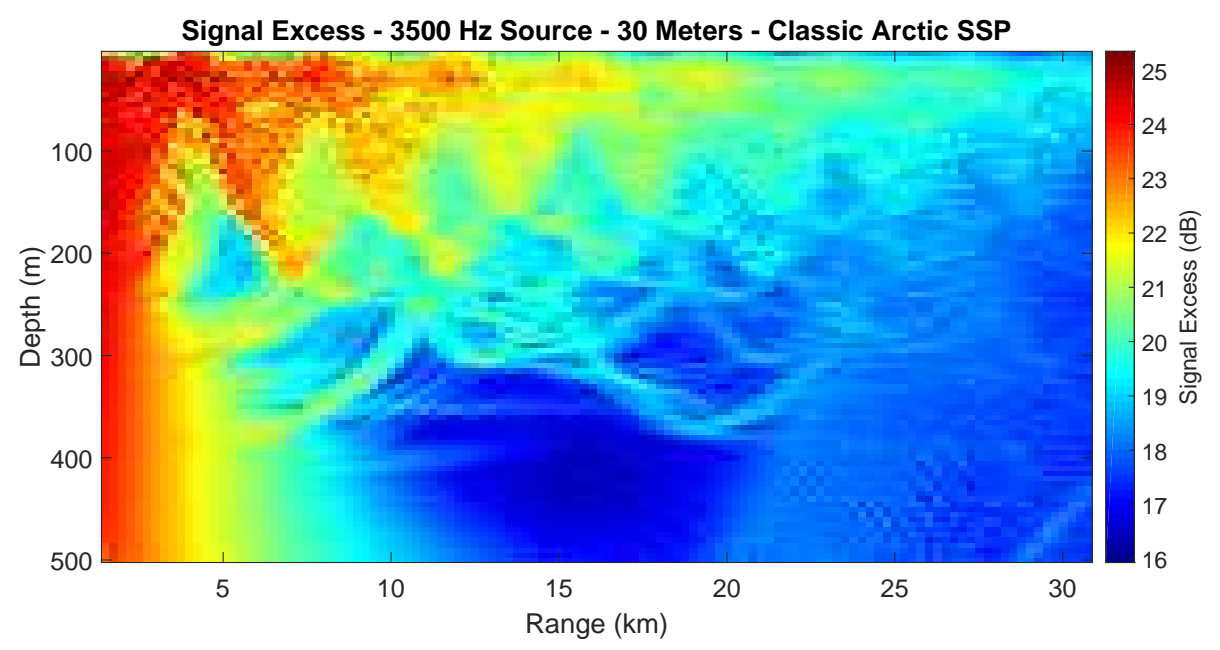

Figure 4.24: Signal excess for a $3500 \mathrm{~Hz}$ source at 30 meters in the classic Arctic with smooth ice cover.

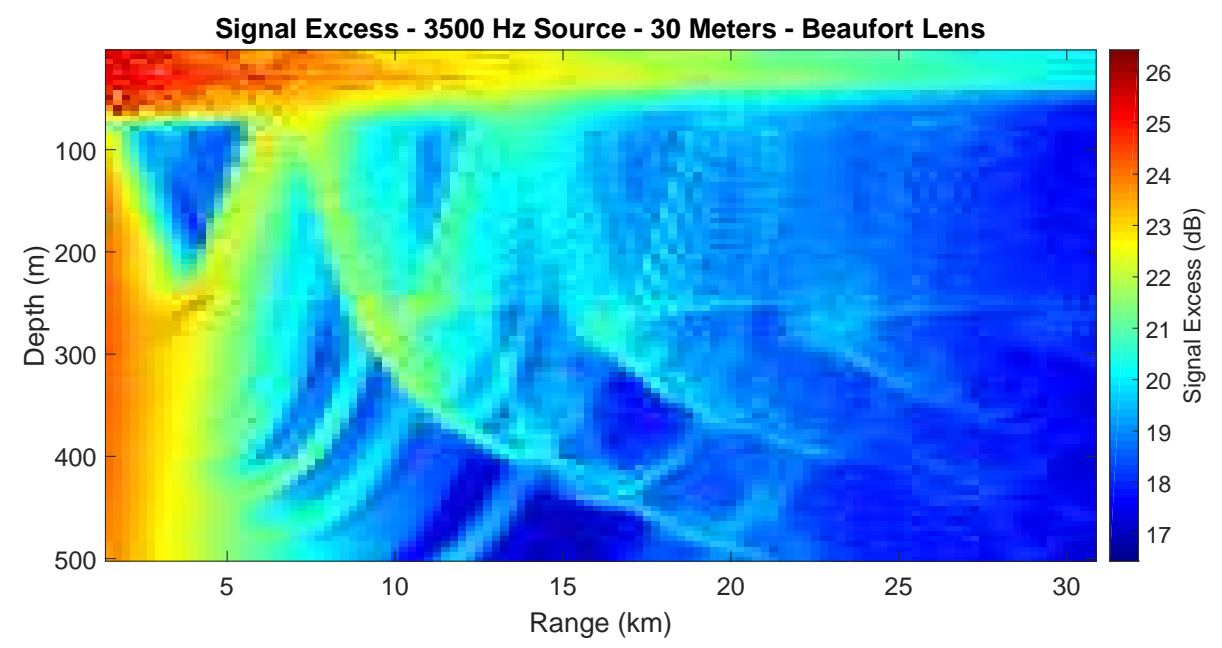

Figure 4.25: Signal excess for a $3500 \mathrm{~Hz}$ source at 30 meters in the presence of the Beaufort Lens with smooth ice cover. Notice the area of very low signal excess from about $75 \mathrm{~m}$ - $225 \mathrm{~m}$ depth at ranges of $1-6 \mathrm{~km}$ known as a shadow zone. This heavy shadow zone caused the acoustic tracking difficulties experienced at ICEX-16 as anything operating in this region would be very difficult to communicate with or track. 
more similar to the $300 \mathrm{~Hz}$ pulse.

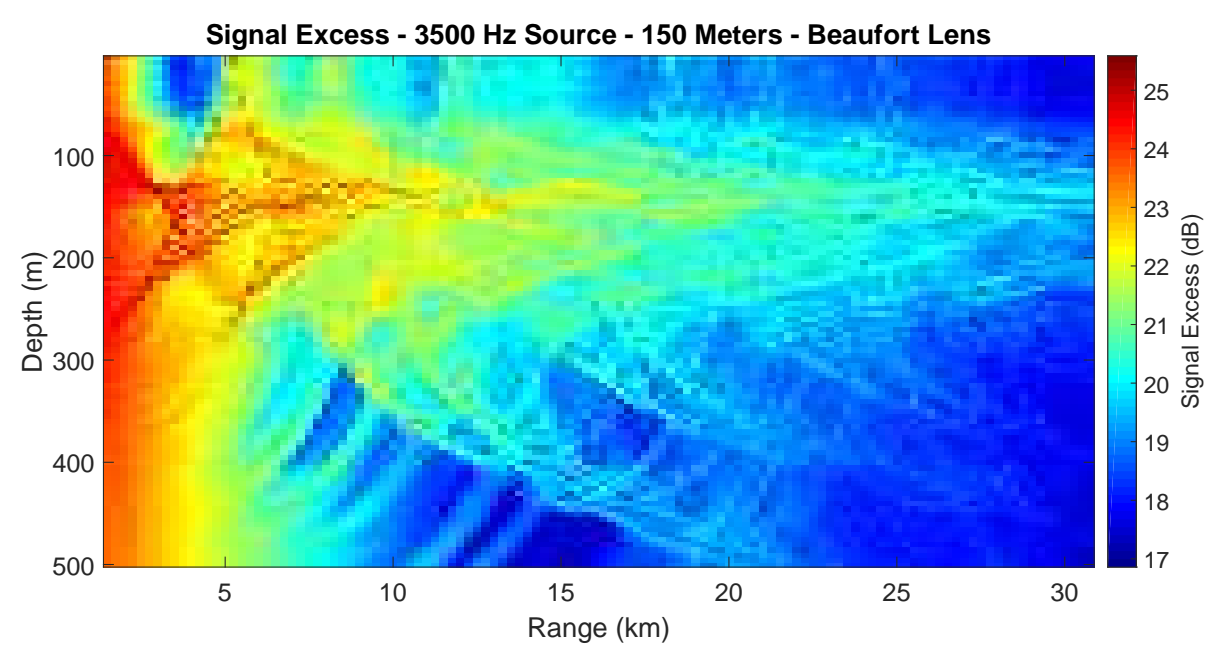

Figure 4.26: Signal excess for a $3500 \mathrm{~Hz}$ source at 150 meters in the presence of the Beaufort Lens with smooth ice cover.

Figure 4.27 shows the difference in signal excess between a shallow source and a deep source. At ranges out to 30 kilometers, there is about a 4 $\mathrm{dB}$ increase in signal excess in the duct. As expected, there is a decrease in signal excess in the surface duct when the source is moved out of the surface duct. Below the duct, there is not a significant change between a shallow and deep source.

Once again, the initial analysis assumes depth independent noise which is not a very accurate assumption. The effect of the Beaufort Lens on higher frequency noise is similar to its effect in the $200-400 \mathrm{~Hz}$ range. More noise is trapped in the surface duct resulting in a higher noise power near the surface, and less noise in the duct itself. These results are shown in figure 4.28 and produced with the OASES OASN module.

With the depth dependent noise, the structure of the duct is enhanced 


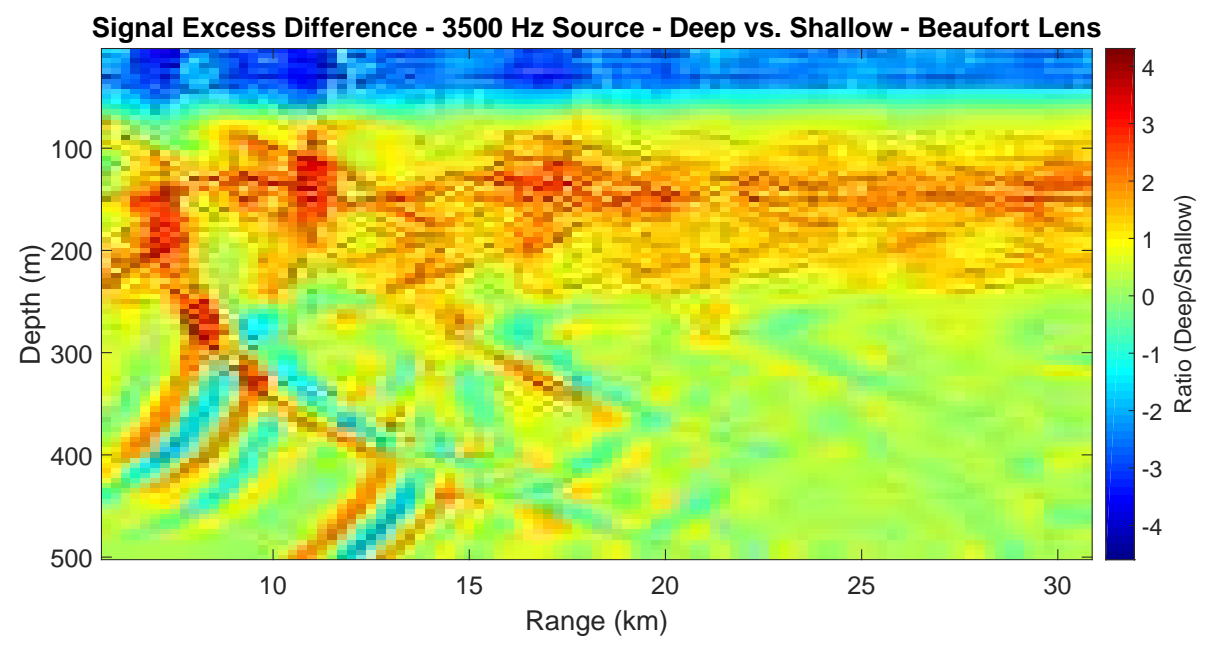

Figure 4.27: Signal excess difference between a $3500 \mathrm{~Hz}$ source at 30 meters and 150 meters in the presence of the Beaufort Lens with smooth ice cover.

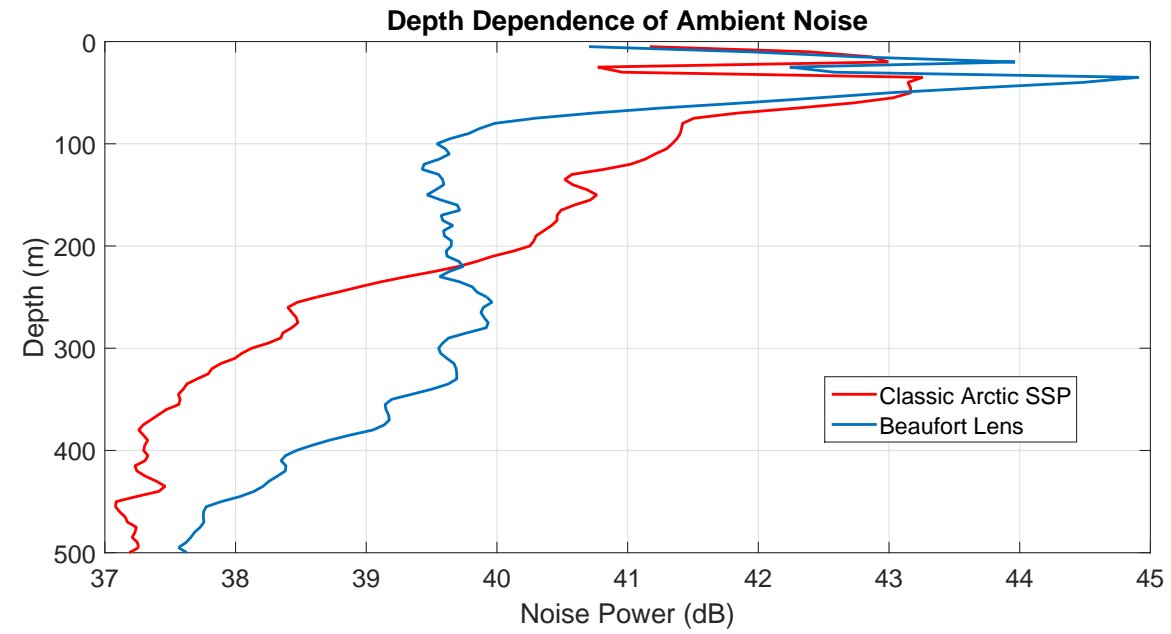

Figure 4.28: Noise power vs. depth for a $3500 \mathrm{~Hz}$ source as predicted by OASN. 
by the increased ducting of the ambient noise in the surface duct. The lower signal excess near the surface and higher signal excess in the duct are apparent in figure 4.29, especially when compared to figure 4.26. The signal excess below the duct is also higher in figure 4.29 than it is with noise that is uniform in depth because of how most noise in the Arctic comes from the surface and high frequency noise attenuates rapidly.

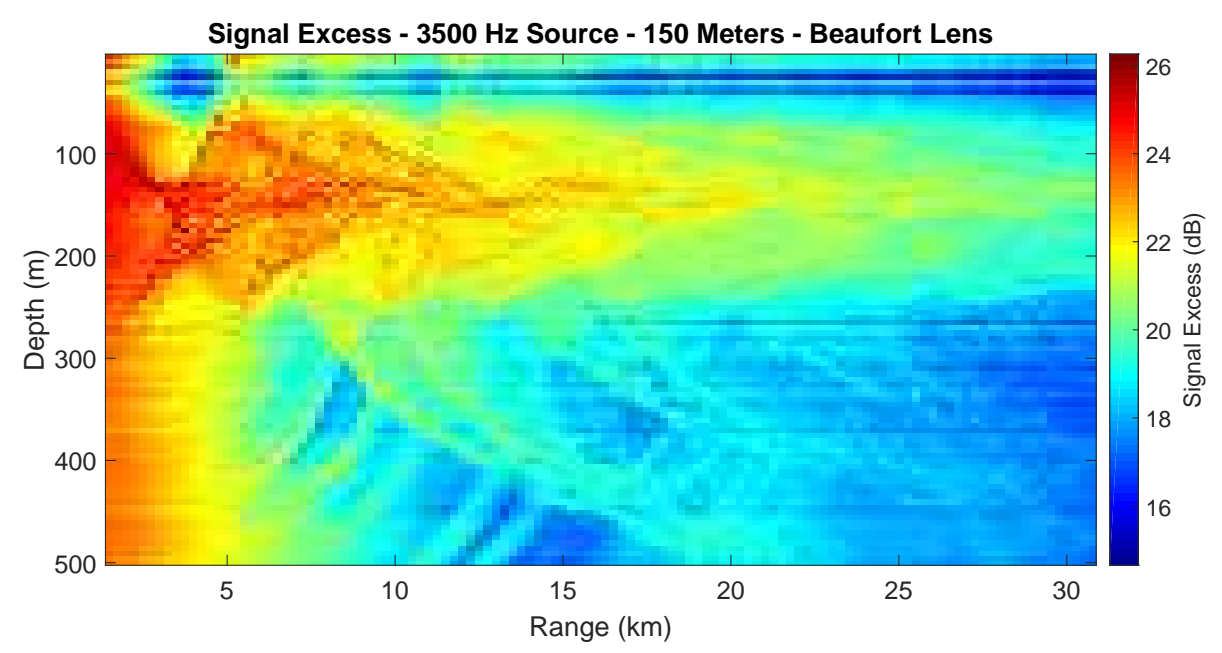

Figure 4.29: Signal excess for a $3500 \mathrm{~Hz}$ source with depth dependent noise in the Beaufort Lens with smooth ice cover. 


\subsection{Channel Capacity}

\subsection{1 $300 \mathrm{~Hz}$ Pulse}

The same $300 \pm 100 \mathrm{~Hz}$ source is used to investigate the effect of the Beaufort Lens on channel capacity. As with section 4.2, the noise is initially assumed to be uniform with depth to accentuate the effect of the Lens on the channel. The Capacity/Bandwidth $(\mathrm{C} / \mathrm{W})$ values of these plots may seem a bit high, but is validated by the high signal excess values (in excess of 20 dB) shown for the sources analyzed.

Figure 4.30 shows the channel capacity estimate for the classic Arctic SSP with a source at 30 meters. The structure is consistent with expectations for an upward refracting SSP as sound is trapped in the upper layers of the water column and is very similar in structure to figure 4.16.

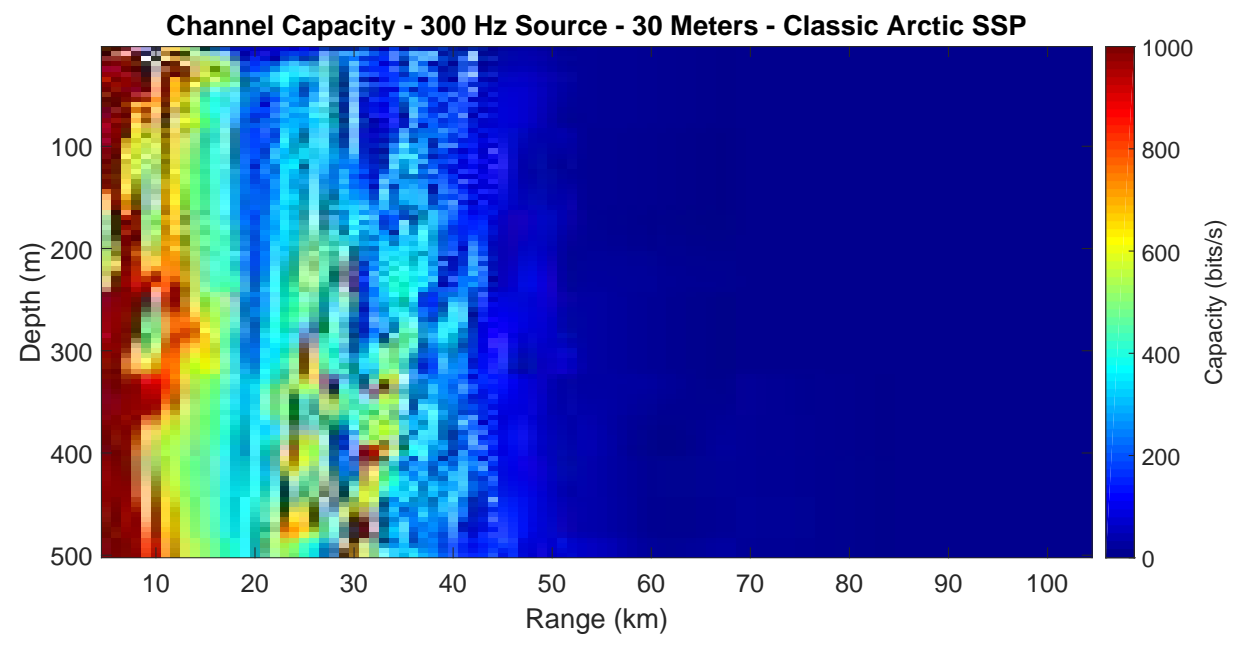

Figure 4.30: Channel capacity estimate using the historic Arctic SSP with a $300 \mathrm{~Hz}$ source at 30 meters and ice cover. The color scale is selected to enhance the gradient as range increases, although it does result in some saturation at ranges less than 10 kilometers. 
Figure 4.31 shows the capacity estimate for the new Arctic SSP for a shallow source. Again, the structure of the channel capacity is very similar to the corresponding signal excess plot, figure 4.17.

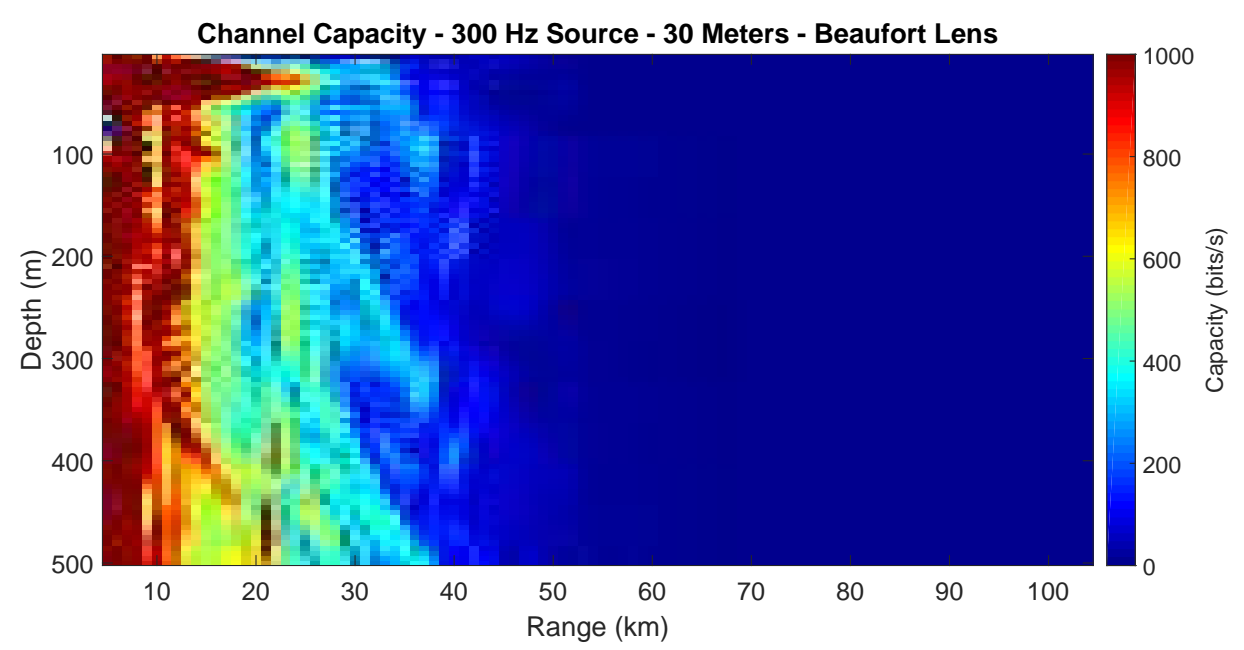

Figure 4.31: Channel capacity estimate using the new Arctic SSP with a 300 $\mathrm{Hz}$ source at 30 meters and ice cover. The surface duct increases the capacity near the surface out to about 20 kilometers, but otherwise the capacity quickly decreases after a range of 30 kilometers as it does in the classic Arctic SSP.

One way to achieve greater channel capacity without forcing the receiver to operate in the surface duct where the ice canopy may be a hazard, is to lower the source depth into the lower duct. Figure 4.32 shows the effect of lowering the source to 150 meters. Here, the Lens is insonified which greatly enhances the channel capacity from about 100-300 meters. This depth band includes common operating depths of many underwater vehicles. Figure 4.33 is included to emphasize the capacity enhancement that lowering the source generates by plotting the difference between the capacity with the source at 30 meters and with the source at 150 meters, $C_{150 m}-C_{30 m}$. Like the signal 
excess plots over-emphasizing scattered energy, capacity is very difficult to achieve in shallow depths due to the complicated multi-path arrival patterns that require precise environmental knowledge to decipher. Therefore, the decrease in capacity at the shallow depths by lowering the source into the duct has very little effect in practice.

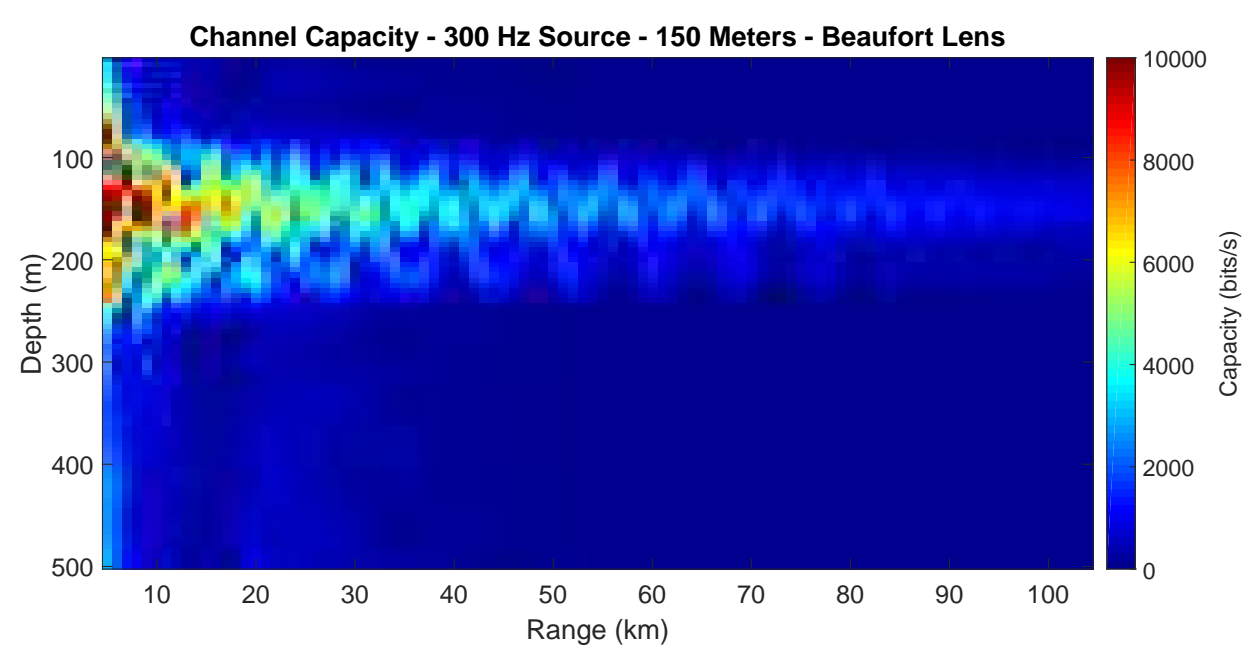

Figure 4.32: Channel capacity estimate using the new Arctic SSP with a 300 $\mathrm{Hz}$ source at 150 meters and ice cover. It is important to note the change in scale of the color bar from the previous two channel capacity plots. Even at ranges of 100 kilometers, the channel capacity is still approximately 1000 $\mathrm{bits} / \mathrm{s}$ in the duct.

With a source power of $193 \mathrm{~dB}$ re $1 \mu P a^{2}$, adding the depth structured noise has little effect because the source level is already so much larger than the ambient noise power. The plot of channel capacity with depth dependent noise is not included, but is very similar to figure 4.32 . 


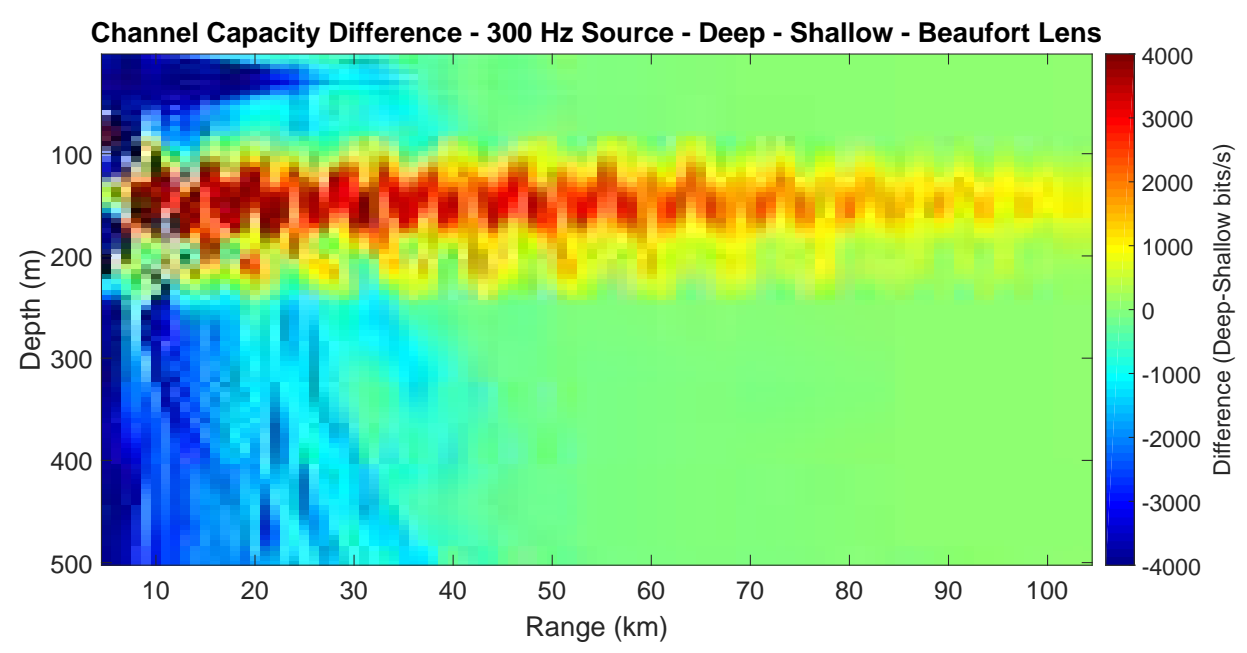

Figure 4.33: The difference between the capacity of the new Arctic with a source at 150 meters to that with a source at 30 meters with ice cover. Here the red shows the enhanced channel capacity in the Beaufort Lens by lowering the source. Lowering the source into the duct increases channel capacity in the duct by over 1000 bits/s at ranges of 100 kilometers, and in excess of $3000 \mathrm{bits} / \mathrm{s}$ from 20 to 40 kilometers. 


\subsection{2 $3500 \mathrm{~Hz}$ Pulse}

As with the lower frequency pulse, the structure of the $3500 \pm 500$ $\mathrm{Hz}$ pulse channel capacity plots very closely mirrors the corresponding signal excess plots. Figure 4.34 shows the channel capacity estimate in the classic Arctic environment. It is important to recall that the ice cover is modeled without roughness for the $3500 \mathrm{~Hz}$ source.

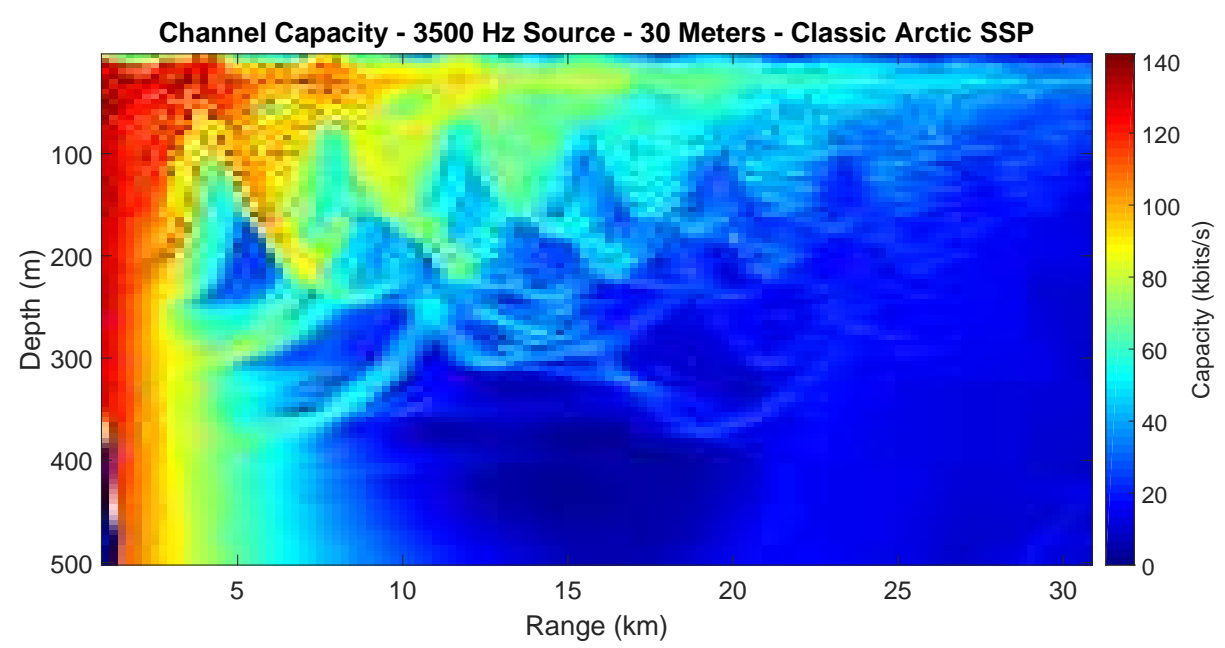

Figure 4.34: Channel capacity estimate for a $3500 \mathrm{~Hz}$ source at 30 meters in the classic Arctic with smooth ice cover. The color scale is now in kilobits/s because of the larger bandwidth of the $3500 \mathrm{~Hz}$ source.

The Beaufort Lens strengthens the surface duct. With the source at 30 meters, the capacity increases near the surface, but decreases outside of the surface duct when compared to the historic profile. While the theoretical channel capacity increases, the ability to actually achieve capacity with an operational communications scheme may not improve because of the challenge of deciphering the modal dispersion and multi-path arrivals as shown in section 4.1. Figures 4.35 and 4.36 highlight the effect of the Beaufort Lens 
on channel capacity as well as the importance of placing the source in the lower duct.

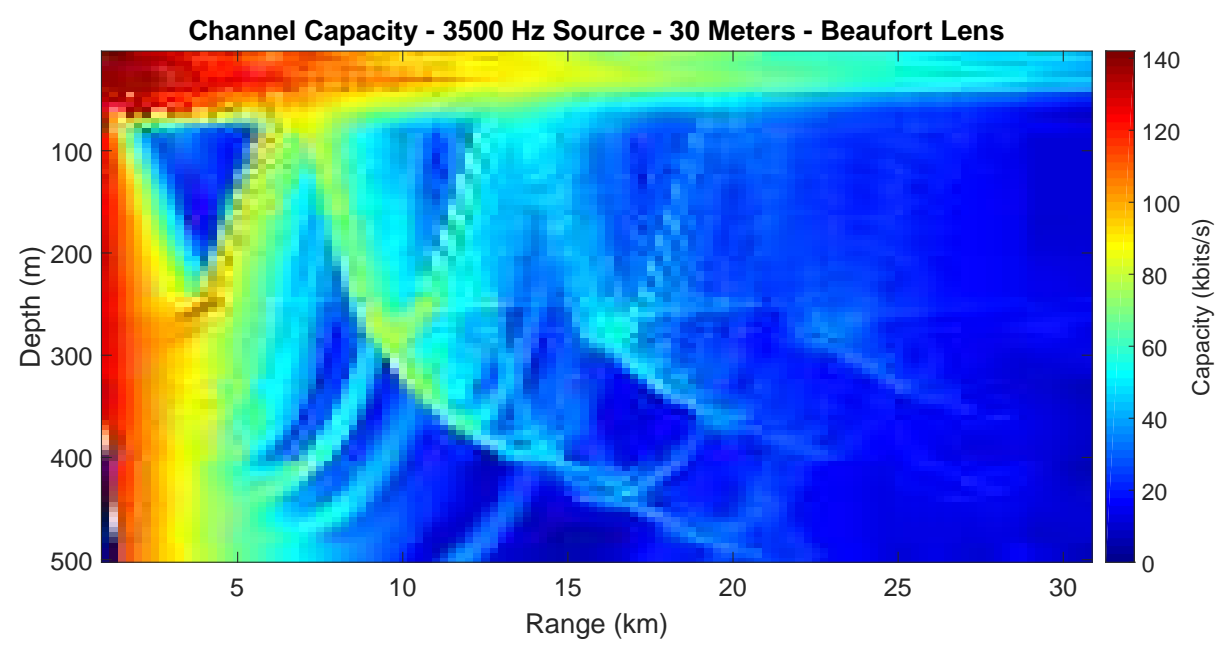

Figure 4.35: Channel capacity estimate for a $3500 \mathrm{~Hz}$ source at 30 meters in the presence of the Beaufort Lens and smooth ice cover. As with the signal excess plot, the heavy shadow zone from 1-6 kilometers and depths of 100-250 meters that caused the tracking problems in ICEX-16 is clearly visible.

Figure 4.37 is included to emphasize the depth dependence of channel capacity. With the channel capacity calculations it is important to remember that the theoretical channel capacity is not always easily achievable in practice. However, by placing the source in the lower duct, the channel capacity increases by a factor of three at ranges of 30 kilometers which dramatically improves acoustic communication capabilities. Additionally, the channel capacity in the heavy shadow zone that was created with the source in the surface duct is significantly improved. 


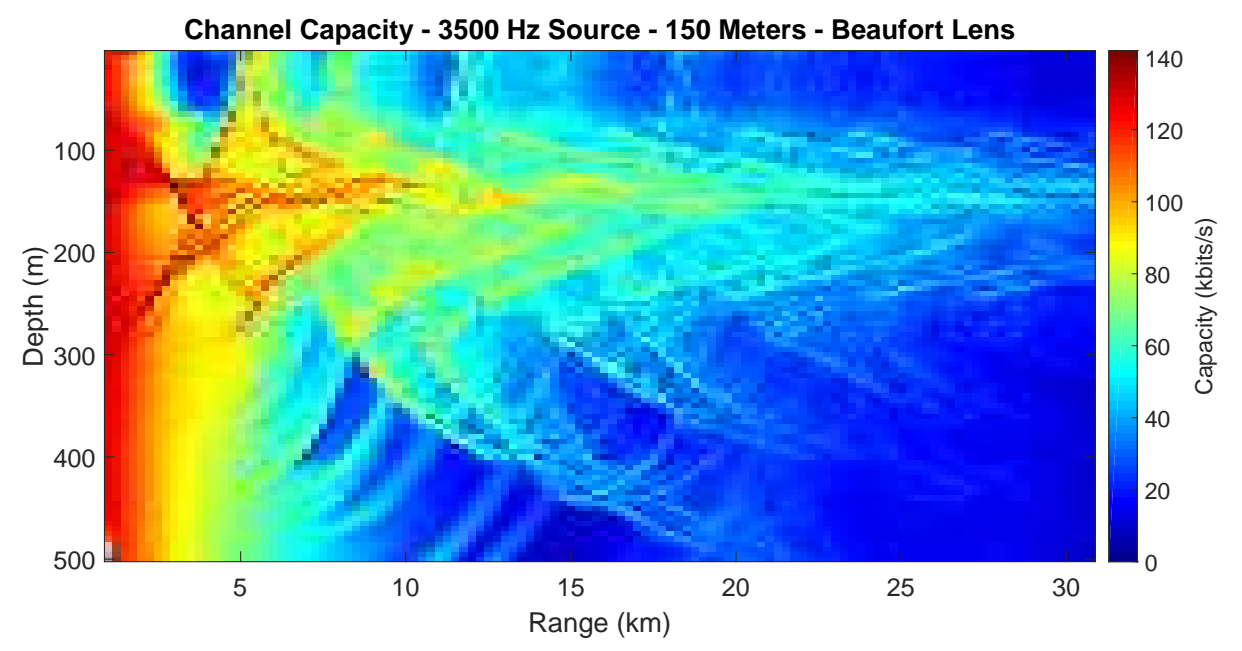

Figure 4.36: Channel capacity estimate for a $3500 \mathrm{~Hz}$ source at 150 meters in the Beaufort Lens and smooth ice cover. 


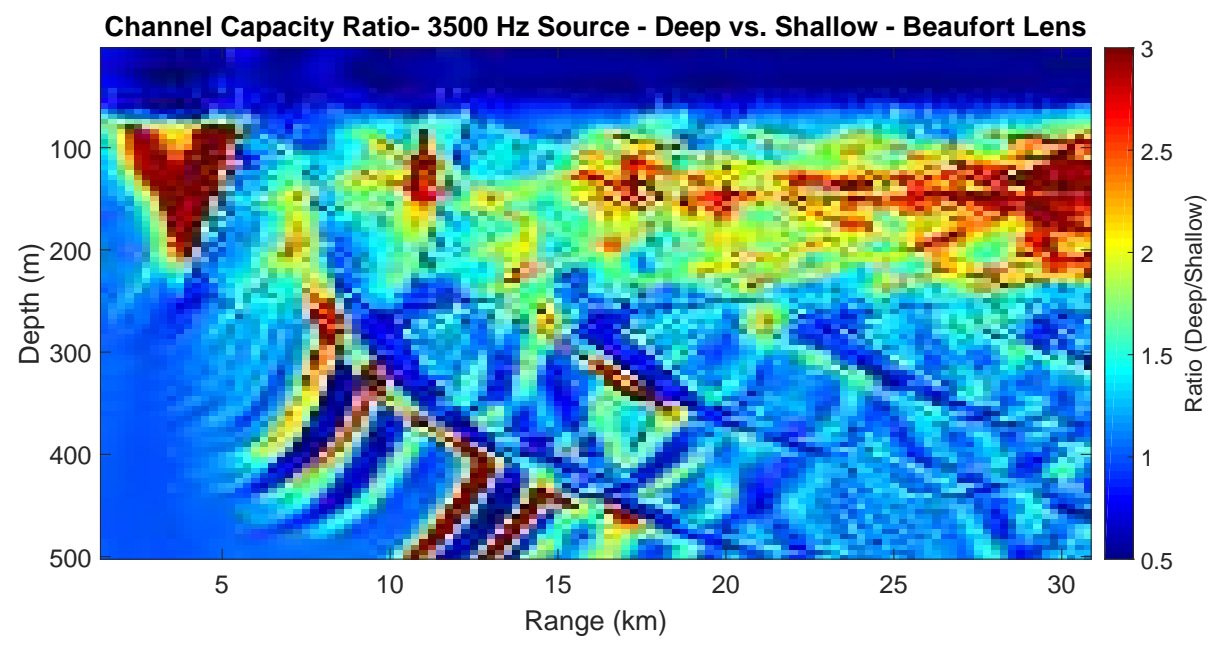

Figure 4.37: The ratio of the capacity in the new Arctic with a $3500 \mathrm{~Hz}$ source at 150 meters to that with a source at 30 meters. Here the red shows the enhanced channel capacity in the Beaufort Lens that is achieved by lowering the source. At ranges in excess of 25 kilometers, the capacity in the Lens increases by a factor of 3. Meanwhile, the capacity in the surface duct is reduced by factor of 2 , but it is unlikely that the theoretical capacity could be achieved in the surface duct because of the complicated arrival patterns. Channel capacity is also enhanced along the ray paths that are able to leak out of the lower duct when the source is at 150 meters. 


\section{Chapter 5}

\section{Conclusion \& Future Work}

The results show two major effects of the duct that can be exploited to enable long range active acoustic transmissions in the Arctic. The modal analysis presented in section 4.1 shows that the ducted modes dominate the signal over long ranges when both the target and the source are in the duct. The ducted modes, even when there are as many as 50 as with the 3500 $\mathrm{Hz}$ pulse, all have very close group speeds which results in minimal modal dispersion, even at long ranges. As the target moves out of the duct, the dispersion and clutter becomes significant and limits both active sonar and communication capabilities.

The second advantage of the duct is that it provides a refracted/refracted waveguide that allows for long range propagation with cylindrical spreading as opposed to spherical. The boundary interaction free path creates high signal excess at long ranges when compared with the classical Arctic SSP. Sections 4.2 and 4.3 highlight this effect of the duct.

The overall effect is that the Beaufort Lens creates a signal with both 
high SNR and minimal modal dispersion when the source is lowered into the duct. These two effects will allow for active sonar or acoustic communications applications at ranges an order of magnitude better than were possible in the classic Arctic environment.

In addition to active sonar and acoustic communications applications, the Beaufort Lens has a direct impact on submarine operations in the Arctic Ocean. The Lens enables long range propagation of low frequency sound at operating depths of submarines. Submarine operating doctrine should be adjusted to leverage the duct for long range tracking of targets when in the Beaufort Sea. Similarly, submarines should avoid the duct to avoid detection or evade incoming torpedoes. Torpedo capabilities will also be impacted by the Beaufort Lens and the Navy should perform a more thorough analysis of how to overcome the new challenges posed by this environment.

Future work will be to validate the models with actual field implementation of a low frequency communication or active sonar system. Opportunities for such experiments exist in future Navy sponsored exercises such as ICEX-18. Additionally, a more robust analysis of Arctic ambient noise should be added to this work. Relatively simplistic assumptions were made about the noise characteristics for this work and depth dependent, correlated noise would provide a more accurate analysis. Model improvements could be made, especially by incorporating under-ice roughness for the higher frequency pulses. Implementing arrays and beamforming in the model would likely enhance the beneficial effects of the Beaufort Lens and is the next logical step in this analysis. Finally, an investigation into different pulse types and shapes and how the Beaufort Lens affects them would be 
extremely valuable before any actual systems are implemented.

The Arctic is an especially dynamic environment as the climate changes. Growing global interest in the region requires a better understanding of the environmental processes that are occurring and how they affect operating in the Arctic Ocean. It is critical that new environmental phenomenon like the Beaufort Lens are not only detected, but leveraged to enhance operating capabilities of the scientific, commercial, and military sectors. 


\section{Bibliography}

[1] F. B. Jensen et al., Computational Ocean Acoustics, 2nd ed. New York, NY: Springer, 2011.

[2] A.G. Litvak, "Acoust. of the Deepwater Part of the Arctic Ocean and of Russia's Arctic Shelf," Herald of the Russian Academy of Sci., vol. 85, no. 3, pp. 239-250, 2015.

[3] T. R. Howe, "A Modal Anal. of Acoust. Propagation in the Changing Arctic Environment", M.S. Thesis, Dept. Mech. Eng., Massachusetts Inst. of Technology, Cambridge, 2015.

[4] Ice-Tethered Profiler Program. Woods Hole Oceanographic Institution. http://www.whoi.edu/itp. Accessed 09 October, 2016.

[5] P. N. Mikhalevsky et. al, "The Transarctic Acoust. Propagation Experiment and Climate Monitoring in the Arctic" IEEE J. Ocean. Eng., vol. 24, no. 2, pp.183-201, Apr. 1999.

[6] W. H. Munk et. al, Ocean Acoustic Tomography, Cambridge, UK: Cambridge University Press, 1995.

[7] R. Courant et. al, Methods of Mathematical Physics, New York, NY: Interscience Publishers, 1989.

[8] T. M. Cover et al., Elements of Information Theory, 2nd ed. Hoboken, NJ: Wiley and Sons Inc., 2006. 
[9] G. M. Wenz, "Acoustic Ambient Noise in the Ocean: Spectra and Sources" J. Acoustical Society of America, vol. 34, no. 12, pp. 19361956, Dec 1962.

[10] H. Schmidt, "OASES Version 3.1 User Guide and Reference Manual," March 11, 2011.

[11] The Ocean Acoustics Library. http://oalib.hlsresearch.com. Accessed 15 September, 2016.

[12] M. B. Porter, "The KRAKEN Normal Mode Program," Washington, DC: Naval Research Laboratory, May 22, 1992.

[13] T. J. Hayward et al., "Underwater Acoust. Commun. Channel Capacity: A Simulation Study," AIP Conference Proceedings 728, 114, 2004. 